\title{
11 Who Gets How Much in Which Relation? A Flexible Theory of Profit Splits in Networks and its Application to Complex Structures
}

\begin{abstract}
Starting from exogenously given negotiation networks, sociological exchange theories explain bilateral divisions of fixed surpluses (e.g., cake, dollar) as consequences of the respective partners' structural embeddedness. There are many competing theories, most of which are, despite of their differences, exclusively concerned with explaining outcomes in pure negatively connected networks with a oneexchange rule. That is, the modelling efforts are directed towards scenarios in which an actual exchange in one relation prevents transfers in other relations and, in addition, only one exchange per round is allowed. Such a narrow focus seems unnecessary since experimental results for networks with more complex relational characteristics are available: (i) networks with positively connected negotiation ties (Yamagishi, Gillmore, and Cook 1988), (ii) networks with varying cake sizes to be partitioned (Bonacich and Friedkin 1998), and (iii) networks where positions differ with respect to the number of exchanges they need to complete (Lovaglia et al. 1995; Skvoretz and Willer 1993; Willer and Skvoretz 1999). In this paper we present a simple model which is not limited to a particular class of exchange networks but allows for point predictions in networks with (all combinations of) complex characteristics. Specifically, we combine the generalized Nash bargaining solution from cooperative game theory with the assumption that both relational features and network positions affect exchange outcomes. Its predictions correspond closely with experimental results.
\end{abstract}

\subsection{Introduction}

Sociological exchange theories seek to explain the bilateral division of a fixed and perfectly divisible surplus (e.g., cake, dollar). Such a positive surplus may, for instance, materialize if the buyer's willingness to pay exceeds the seller's reservation price. Exchange theories then assume that ac- tors agree on the partitioning of the surplus in bilateral negotiations. And, exchange theorists agree with Emerson (1972, 1981) that negotiations and exchange rarely occur in isolate encounters. They thus consider situations in which a given network structure defines matches between pairs of bargaining partners (Cook et al. 1983) and attribute exchange outcomes in dyads to the effects structure has on individual negotiation success. Exchange theory

Thomas Gautschi, University of Mannheim

¿ Open Access. (C) 2020 Thomas Gautschi, published by De Gruyter. (c) BY-NC-ND This work is licensed under a Creative Commons Attribution-NonCommercial-NoDerivatives 4.0 International License.

https://doi.org/10.1515/9783110647495-011 
thus purposely neglects effects other than structure on exchange outcomes (e.g., effects of individual characteristics such as age, sex or education).

In accordance with this assumption, the basic premise of sociological exchange theory is that actors seek to benefit from exchange by exploiting structural advantages. That is, they use their network positions to maximize their individual shares of the cakes to be negotiated on in dyadic bargaining. Theorists thus prefer a rational actor perspective in the sense that interactive choices are characterized by purposive and profit maximizing individual behavior. Consequently, dyadic exchange will produce outcomes in which one actor will not necessarily obtain as much exchange profit as the other. And, exogenous restrictions may force actors to choose an exchange partner from a larger set of negotiation partners. In accordance with these ideas, sociological exchange theory thus searches for answers on two fundamental questions. First, who gets, depending on his position in the negotiation structure, how much of the surplus? And second, who exchanges with whom if, after negotiations with different partners, an exchange partner has to be chosen? Put differently, can structural deviations between the bargaining and exchange networks be identified ${ }^{1}$ Besides the consensus to study the effects of structure only on exchange outcomes, sociological exchange theories have other common features. At least partly, these features reflect that theory building was closely related with the restricted exchange situations studied in laboratory experiments. Most experimental research focuses on the study of exchange structures with negatively connected and equally valued relations. ${ }^{2}$ The latter refers to exchange systems where relations concern the split of an identical surplus. And, negatively connected systems are characterized by the fact that exchange in one relation tends to prevent exchange in other relations (e.g., dating networks). Accordingly, the close relationship between model building and experimental work lead to the situation that the focus was put on developing theories for negatively connected exchange structures only. ${ }^{3}$ Even though most

1 While we do not address the latter question in this paper either, we show in Braun and Gautschi (2007) how our model (or any other model which provides point predictions on the division of cakes) can be used to decide whether given bargaining ties become exchange patterns if there is competition between potential trading partners. However, there are publications which endogenize the formation, stabilization, and modification of actor relations in networks (e.g., Aumann and Myerson 1988; Bala and Goyal 2000; Demange and Wooders 2005; Dutta and Jackson 2003; Gould 2002; Jackson and Wolinsky 1996; Skyrms and Pemantle 2000; Slikker and van den Nouweland 2001; Vega-Redondo 2007; Watts 2001) and thus treat the network as the "explanatory variable".

2 Exceptions are Yamagishi, Gillmore, and Cook's (1988) experiments on a positively connected 5LINE and Bonacich and Friedkin's (1998) experiments on simple exchange structures with variations in the value of the cake to be partitioned. Networks with positively connected exchange ties are normally associated with situations in which resource transfers in one relation tend to promote transfers in other relations (e.g., communication networks).

3 Modifying and extending Coleman's $(1973,1990)$ competitive equilibrium approach, Yamaguchi (1996) is the only theory which can be applied to negatively and positively connected exchange 
established theories can be adjusted to account for variations in cake sizes to be divided (e.g., Friedkin's (1986, 1992) Expected Value theory, Skvoretz and Willer's (1993) Exchange Resistance theory, Yamagishi and Cook's (1992) Equi-Dependence theory, and partly, Markovsky, Willer, and Patton's (1988) GPI measure), the focus of most theories nevertheless appears considerably narrow and seems an unnecessary restriction. Especially since Cook et al. (1983), Cook and Emerson (1978), and Emerson (1972) have established the distinction between negatively and positively connected exchange relations quite some time ago.

On the one hand, as claimed above, modeling efforts are confined by its close connection to the experimental focus on negatively connected exchange structures and equally valued relations (i.e., homogeneity with respect to cake sizes). On the other hand, however, basic characteristics of the experimental bargaining protocol are still not reflected by established theories ${ }^{4}$ such that they are, at least, partly based on divergent assumptions. In short, two points are most relevant. First, all theories assume that negotiation partners pursue their self-interest, and such is stimulated in laboratory experiments. However, the theories usually do not specify an optimization problem. And, it is unclear where network features enter the choice calculus and how they influence decision making. Second, interactive choices characterize (experimental) negotiation processes. However, only a few theories explicitly refer to game-theoretic ideas but none uses a dyadic bargaining solution with a solid foundation in non-cooperative bargaining theory. Not one theory thus reflects the experimentally implemented bargaining protocol of alternating offers. This divergence between experiments and theories seems questionable since experimental results make up the measure rod against which the theories' performance is tested.

structures. He equates negative (positive) connections with closely substitutable (complementary) exchange relations and introduces a flexible continuous parameter for substitutability/complementarity (viz., the elasticity of substitution). Yamaguchi's theory thus embraces situations in which exchange in one relation tends to prevent or promote transfers in others. Its application requires, however, an ad hoc specification of the elasticity of substitution. And, it is limited to the analysis of either substitutable or complementary relations in a given network. Combining basic ideas of his original model with additional assumptions, Yamaguchi (2000) presents a theoretical analysis of structures characterized by the simultaneous presence of both substitutability and complementarity among the multiple exchange relations of an actor.

4 There are a wide range of different theories but about as many controversies among them as well (e.g., Bienenstock and Bonacich 1992, 1993, 1997; Bonacich 1998, 1999; Bonacich and Bienenstock 1995; Bonacich and Friedkin 1998; Burke 1997; Friedkin 1992, 1993, 1995; Lovaglia et al. 1995; Markovsky et al. 1993, 1997; Markovsky, Willer, and Patton 1988, 1990; Skvoretz and Fararo 1992; Skvoretz and Lovaglia 1995; Skvoretz and Willer 1991, 1993; Thye, Lovaglia, and Markovsky 1997; Yamagishi and Cook 1990; Yamagishi, Gillmore, and Cook 1988; Yamaguchi 1996, 1997, 2000). Special issues of journals (cf., Social Networks 14, No. 3-4, 1992 and, at least partly, Rationality and Society 9, No. 1-2, 1997) contain additional articles and controversies. Willer and Emanuelson (2008) test ten prominent theories on their empirical relevance. 
Current sociological exchange theories are limited in scope which restricts them in being able to make predictions for exchange systems with more realistic features. Especially given the empirical accuracy of established theories in predicting outcomes in negatively connected structures, researchers should make the necessary further step, adjusting and revising their theories (or developing new theories) to be able to deal with more complex networks (viz., negatively connected, positively connected and mixed network structures with potentially unequally valued relations and no restrictions on node-specific parameters such as the number of exchanges a position intends to complete). Results from experiments employing networks with more complex relational and positional characteristics are publicly available. Yamagishi, Gillmore, and Cook (1988), for instance, present results for a positively connected 5Line. Positive connections were ensured by (i) introducing distinct resources at opposite ends of the line structure, (ii) dictating that resource $x$ be traded for resource $y$ in one relation and $y$ for $x$ in the other relation, and (iii) by paying profit points only for pairs of resources collected (complementary goods). Moreover, Yamagishi, Gillmore, and Cook correctly emphasize that real networks often are mixtures of both, negatively and positively connected relations. Consequently, they additionally provide experimental results for a mixed network structure. ${ }^{5}$ Experimental evidence of yet another kind is offered by Bonacich and Friedkin (1998). They discuss results from an experiment on four network structures, each of which was characterized by unequally valued relations. As their results show, profit distributions are considerably affected, compared to a system of equally valued relations, by the heterogeneity in the surpluses to be divided in bilateral negotiation. Finally, Skvoretz and Willer (1993) and Willer and Skvoretz (1999) discuss experimental findings from networks with equally valued relations but where positions differed with respect to the number of exchanges they needed to complete. These networks were thus characterized by a heterogeneity in the number of exchanges positions conclude per round of negotiation and exchange. For those structures where results from equivalent networks with a one-exchange rule are available, it is found that dyadic profit splits indeed differ from the simple (homogeneous) scenario.

While there are theories which can, at least partly, deal with some of the more complex network characteristics (e.g., Markovsky et al. 1993; Skvoretz and Willer 1993; Yamaguchi 1996, 2000), there is not a single model which could consistently address all aspects of complex networks. Moreover, some theories which can partly handle complex networks are adjusted versions of existing theories which have been revised such that ad hoc assumptions now delineate their updated versions. This indicates the need for a sufficiently general theory of exchange networks.

5 This relational assessment was ensured by 'adding' two new actors to one of the peripheral positions in the positively connected 5-LiNE. This position can now obtain identical resources (say, $x$ ) through either of his new contacts. Since they offer the same resource, they are thus negatively connected to the former peripheral 5-LINE actor. 
First of all, such a model should be parsimonious and simple to apply. At the same time, if researchers stress that negotiation partners pursue their self-interest (i.e., the rationality postulate stimulated in laboratory experiments), a theory should clarify how structural features affect the actors' decision-making and how their interactive choices determine the negotiation outcomes. Second, a model may not be limited to the analysis of negatively connected settings but must embrace all relational assessments of ties. Third, it should allow for unequally valued relations (i.e., variations in terms of the surplus to be partitioned) and positional heterogeneity in the number of exchanges positions wish to complete per round. Finally, the model should offer unique point predictions for negotiation outcomes which closely fit the available experimental evidence. That is, it should be able to predict empirical evidence from simple as well as from more complex bargaining experiments.

We present and apply a model with these properties in the remainder of this article. This model combines the generalized Nash $(1950,1953)$ bargaining solution from cooperative game theory (cf., Binmore 1992) with a specific definition of each actor's bargaining power in terms of relational as well as positional features and network embeddedness. ${ }^{6}$

\subsection{Relational assessment}

Even though the classification of negatively and positively connected network relations (Cook and Emerson 1978; Cook et al. 1983; Emerson 1972) is widely accepted in the sociological exchange literature, there is no sound and formal definition for negative or positive ties. The lack of such a definition poses no problem in the case of pure negatively or positively connected negotiation structures. However, Yamagishi, Gillmore, and Cook (1988) correctly emphasize that real networks often are mixtures of both types of relations (e.g., exchange in the Kula Ring as described by Malinowski 1922). The loose definition of negatively and positively connected ties - that is, exchange in one relation either prevents or promotes transfers in others - is unfortunately not sufficient for a precise mathematical description of mixed negotiation networks. Consequently, unique profit point predictions for mixed networks are not necessarily guaranteed. From a methodological point of view it is, however, essential

\footnotetext{
6 Since we first presented this basic idea (Gautschi 2002), other authors also suggested exchange theories based on the Nash bargaining solution (Bayati et al. 2015; Chakraborty and Kearns 2008; Chakraborty et al. 2009; Kleinberg and Tardos 2008). The cooperative bargaining solution coincides with the subgame-perfect Nash equilibrium outcome of Rubinstein's game if the focus is on the limiting scenario in which the amount of time between proposals vanishes (cf., Binmore 1985, 1998; Muthoo 1999; Osborne and Rubinstein 1990). The generalized Nash bargaining solution thus is an appropriate cooperative solution concept because it can be derived from a strategic analysis in the sense of non-cooperative game theory.
} 
to have a definition of negative and positive connection which allows for an unequivocal characterization of network ties. Only this provides a basis for unique profit point predictions in all types of networks.

In experiments, positive connections are ensured by demanding that two goods flow through the network (Yamagishi, Gillmore, and Cook 1988). ${ }^{7}$ Since actors can derive utility from pairs of goods only - that is, goods which are always consumed together in fixed proportions (perfect complements) -, they need to collect both goods in equal quantities. Exchange for one good in one relation thus promotes transfers in other relations providing the complementary good. Each actor will engage in a series of dyadic bargaining sessions to obtain as much as he can from each good. However, this procedure is not suited to define mixed networks in a proper sense.

To eliminate this problem, we introduce a new and more precise classification of network connections which, however, embraces pure negatively and pure positively connected network structures as special cases. It is thus ensured that profit point predictions can be compared with empirical evidence stemming from laboratory experiments on negatively and/or positively connected networks. However, it eliminates, on the one hand, the troublesome correspondence between negative connections and the concern for the distribution of just one homogeneous good. And, on the other hand, it abandons the correspondence between positive connections and the pairwise distribution of perfectly complementary goods. The new classification hinges on three fundamental network characteristics. ${ }^{8}$

Before we can define the new relational types, we thus need to introduce these basic network parameters. In any given bargaining network, we can distinguish between peripheral and non-peripheral positions. Specifically, actor $i$ is said to be at a peripheral network position if he has just one bargaining partner (i.e., $n_{i}=1$ ). Otherwise, actor $i$ is said to be at a non-peripheral position if he has two or more distinct bargaining partners (i.e., $n_{i} \geq 2$ ).

In addition, we introduce two node-specific parameters for the precise classification of relations. Specifically, let $m_{i}$ be the number of actor $i$ 's bargaining relations (i.e., $m_{i}$ is the number of (non-directed or symmetric) arcs associated with node $i$ ) and let $g_{i}$ be the number of exchanges actor $i$ intends to complete (i.e., $g_{i}$ could denote the

7 Note, however, that positive connections do not necessarily involve distinct goods. Exchanges between, say, a professional athlete and his agent as well as complementary relations between the agent and organizers of athletic events may involve money only. Or, a broker can obtain information in one relation which eventually makes it possible to give advice to another of his partners. However, neither of these alternative considerations on positively connected ties solves our methodological problem.

8 Willer and Skvoretz (1999) also present an alternative categorization of network connections which, as they emphasize, differs from the positive/negative classification. Their distinction embraces five types of connections. It draws on the ranking and values of several node-specific parameters which are, however, at least partly, unique to their theoretical approach. 
number of goods $i$ wants to buy or sell in a period), where $m_{i} \geq g_{i} \geq 1{ }^{9}$ For any actor $i$ in a given network, it thus will either hold $m_{i}>g_{i} \geq 1$ or $m_{i}=g_{i} \geq 1$. These cases refer to the following situations:

- If $m_{i}>g_{i} \geq 1, i$ has more bargaining ties than he can conclude exchanges. Actor $i$ therefore has a set of competing bargaining ties with respect to the exchange of one or more substitutable goods. He can thus select his exchange partner(s) from his set of exchange relations.

- If $m_{i}=g_{i} \geq 1, i$ can conclude just as many exchanges as he has bargaining partners. We say that $i$ has non-competing bargaining relations. Consequently, there are two possibilities: $m_{i}=g_{i}=1$ or $m_{i}=g_{i}>1$. When $m_{i}=g_{i}=1$ is given, $i$ has just one bargaining relation with respect to an exchange of one specific good he intends to complete. On the other hand, if $m_{i}=g_{i} \geq 2$ is satisfied, $i$ has different non-competing bargaining relations with respect to the exchange of two or more goods which are either complementary or independent (i.e., neither complementary nor substitutable). ${ }^{10}$

Taking into account that an actor may either be located at a peripheral position or at a non-peripheral position, these above cases provide the basis for the new relational classification of an individual $i$ who is matched with a bargaining partner $j$ :

Rival Orientation: $i$ has a rival orientation in the relation with $j$ if

- $i$ has a non-peripheral position $\left(n_{i} \geq 2\right)$ and competing bargaining ties from which he can select exchange relations $\left(m_{i}>g_{i} \geq 1\right)$, or,

- $i$ is at a peripheral position $\left(n_{i}=1\right)$ and his only partner $j$ has competing bargaining relations $\left(m_{i}=g_{i} \geq 1\right.$ and $\left.m_{j}>g_{j} \geq 1\right)$.

Non-Rival Orientation: $i$ has a non-rival orientation in the relation with $j$ if

- $i$ has a non-peripheral position $\left(n_{i} \geq 2\right)$ and non-competing bargaining ties $\left(m_{i}=g_{i} \geq 2\right)$, or,

- $i$ is at a peripheral position $\left(n_{i}=1\right)$ and his only partner $j$ has non-competing bargaining relations $\left(m_{i}=g_{i} \geq 1\right.$ and $\left.m_{j}=g_{j} \geq 2\right)$.

Our postulate reflects that people decide about the type of their connections in reality we assume that every system actor who has two or more ties in the given negotiation network classifies each of his relations as either a rival or a non-rival connection. This

9 Our postulate reflects that these parameters denote decisions and/or restrictions people take or face in reality. However, in experimental work the parameters $m_{i}$ and $g_{i}$ are exogenously fixed by the experimenter, together with the relations defining the bargaining structure.

10 Note that here lies a major difference between our classification and the negative/positive connection classification. A positive connection is generally identified with perfectly complementary goods. Our classification is flexible enough to embrace situations in which the analogous of a positively connected tie does not necessarily ask for complementary goods to be exchanged. 
typology further draws on the assumption that an actor with just a single bargaining partner (i.e., an actor at a peripheral position) in a network with two or more relations simply adjusts to the relational categorization of his only partner (in the special case of the Dyad-structure, each actor has a peripheral position and, by postulate, a non-rival orientation). ${ }^{11}$ To keep things as simple as possible, however, the actors' assessments of relations are exogenous components in our model. ${ }^{12}$

Combining the possible cases of rival and non-rival orientations for two adjacent actors $i$ and $j$ in any exogenously given bargaining network suggests a parsimonious classification of relations:

Pure Rival Connection: A pure rival relation between $i$ and $j$ exists if both actors have a rival orientation. ${ }^{13}$

Pure Non-Rival Connection: A pure non-rival relation between $i$ and $j$ exists if both actors have a non-rival orientation. ${ }^{14}$

As a consequence of our assumptions, a peripheral actor never will be involved in a mixed relation. When an actor $i$ has two or more partners, however, he may face a non-peripheral bargaining partner $j$ with another relational orientation. If so, a mixed relation between $i$ and $j$ will exist. ${ }^{15}$

Mixed Connections: A mixed relation between $i$ and $j$ exists if the actors differ in terms of their relational orientation.

Mixed Rival Connection: A mixed rival relation is said to exist if $i$ has a rival orientation and $j$ has a non-rival orientation.

Mixed Non-Rival Connection: A mixed non-rival relation is said to exist if $i$ has a non-rival orientation and $j$ has a rival orientation.

11 Note that this assumption does not exclude that the individual classifications of network connections simply reflect systemwide incentives. Our postulate therefore should not create problems in experimental work as long as test persons (have learned to) systematically react to incentives. Suppose, in accordance with the usual design of network exchange experiments (e.g., Skvoretz and Willer 1991), that subjects' monetary compensation for participation explicitly depends on their bargaining success. We expect that these incentives ensure, at least after several training rounds, relational assessments in the sense of the experimenters.

12 Following the usual practice in the field, we thus predict exchange profits and structures only. This clearly simplifies the model - apart from the actors' structural positions (e.g., just one potential exchange relation or several bargaining relations), the assessments of relations may reflect the network type (e.g., dating or communication network), the number and type of goods to be divided, and the existence of systemwide restrictions (e.g., laws, norms, rules).

13 If the bargaining network is characterized by $m_{i} \geq g_{i}=1$ for all $i=1,2, \ldots, n$, the structure of pure rival connections corresponds to what is normally referred to as a pure negatively connected network (with a one-exchange rule).

14 The only experimental work on a positively connected network structure is due to Yamagishi, Gillmore, and Cook (1988). This bargaining network was characterized by $m_{i}=g_{i}=2$ for all nonperipheral actors, and thus constitutes a special case of a pure non-rival network.

15 Under special parameter constellations, these mixed relations correspond to negatively-positively and positively-negatively connected bargaining structures, respectively. 
More precisely, there is a mixed rival connection if $n_{i} \geq 2$ and $m_{i}>g_{i} \geq 1$ as well as $n_{j} \geq 2$ and $m_{j}=g_{j} \geq 2$, but a mixed non-rival connection if $n_{i} \geq 2$ and $m_{i}=g_{i} \geq 2$ as well as $n_{j} \geq 2$ and $m_{j}>g_{j} \geq 1$. In all other cases, the relational orientations of two bargaining partners coincide such that they face either a pure rival or a pure non-rival connection.

Consider the example of the basic T-Shape structure where position $\mathrm{B}$ is connected to two peripheral actors, $A_{1}$ and $A_{2}$, as well as to position $C$. The latter is in addition tied to the peripheral position D. Assume further that we seek to ensure mixed relations by inserting complementary goods at both ends. Assume that the peripheral A's are provided with good $a$ and the peripheral position $\mathrm{D}$ with good $d$. According to Yamagishi, Gillmore, and Cook (1988), the B-A ties is negatively connected - the A's are competitors in providing B with good $a$ - while the $\mathrm{B}-\mathrm{C}$ and $\mathrm{C}-\mathrm{D}$ relations are positively connected. The theoretical definition of negative and positive ties, however, creates difficulties in appropriately defining such a mixed network. Our new classification of network connections makes no assumption about whether ties classified on the dyadic level would promote or prevent transfers in other relations. We thus can uniquely define mixed networks. In our example, $\mathrm{B}\left(n_{B}=3, m_{B}=3\right.$, and $\left.g_{B}=2\right)$ as well as the two As $\left(n_{A}=1, m_{A}=1\right.$, and $\left.g_{A}=1\right)$ have rival orientations, due to $m_{B}>g_{B} \geq 2$ and $m_{A}=g_{A} \geq 1$. On the other hand, both $\mathrm{C}\left(n_{C}=2, m_{C}=2\right.$, and $\left.g_{C}=2\right)$ and $\mathrm{D}\left(n_{D}=1\right.$, $m_{D}=1$, and $\left.g_{D}=1\right)$ have non-rival orientations since $m_{C}=g_{C} \geq 2$ and $m_{D}=g_{D} \geq 1$. This results in a T-Shape with mixed connections, where the B:A relations are pure rival connections and the C:D is a pure non-rival connection. The B:C relation is, from B's point of view, a mixed rival connection while $C$ would see the connection as a mixed non-rival one. Consequently, both $\mathrm{B}$ and $\mathrm{C}$ can engage in two exchanges per round while the As as well as D can only exchange once. Since $B$ has three ties but seeks to exchange twice, he can choose between exchanging with $\mathrm{A}_{1}$ and $\mathrm{A}_{2}$, or with $\mathrm{C}$ and either of the As. Actor C, in contrary, is - if two exchanges need to be concluded - forced to exchange with $\mathrm{C}$ and $\mathrm{B}$. Note, however, that the sequence of dyadic exchanges does not matter for our new classification of relations. It therefore allows for a proper definition of a mixed network.

\subsection{Theoretical model}

Consider an exogenously given network with $m$ mutual ties between a finite number of rational actors $(i, j, k=1,2, \ldots, n) .{ }^{16}$ These symmetric relations limit the

16 As will become clear later, the conclusion of the model does not rest on the assumption of fully rational and selfish egoists. As long as an actor's utility is determined by his own share of the cake and the actor purposively negotiates his share of the surplus, it suffices to assume boundedly rational, but learning actors. The use of the rational actors' framework, however, simplifies the presentation of the model. 
matches of potential partners for negotiations and exchanges. Each bargaining session refers, by postulate, to the bilateral distribution of a fixed quantity of a perfectly divisible resource (e.g., money). Exchange appears here, in accordance with sociological approaches (e.g., Bonacich and Friedkin 1998; Willer 1999), as an agreement of two rational actors on the division of a fixed surplus. Specifically, we assume that the actors $i$ and $j$ bargain over the partition of a surplus of given value $v_{i j}=v_{j i}$. When $x_{i j}$ represents $i$ 's negotiated share of the value $v_{i j}$, it holds that $0 \leq x_{i j} \leq$ $v_{i j}{ }^{17}$ Put differently, $x_{i j}$ denotes $i$ 's negotiated exchange profit in the relation with $j$.

The profit shares $x_{i j}$ and $x_{j i}$ are to be explained in terms of structure and positional features. For that purpose, it is postulated that, once $i$ and $j$ negotiate over the partition of the value $v_{i j}$, they determine their profit shares as if they would apply the generalized version of the Nash $(1950,1953)$ bargaining solution from cooperative game theory (see, e.g., Binmore 1987, 1992). That is, they choose the profit shares $x_{i j}$ and $x_{j i}$ as if they would solve the optimization problem

$$
\max x_{i j}^{b_{i}} x_{j i}^{b_{j}} \text { subject to } x_{i j}+x_{j i}=v_{i j} \text {, }
$$

where the positive parameters $b_{i}$ and $b_{j}$ refer to $i$ 's and $j$ 's absolute level of individual bargaining power. ${ }^{18}$ As will become clear below, the solution of the optimization problem implies that the split of the given surplus between $i$ and $j$ reflects the combination of their bargaining powers (i.e., the distribution of "relative bargaining power" determines the negotiated exchange profits). And, most important, the generalized Nash bargaining solution has a solid non-cooperative foundation in the limiting equilibrium of Rubinstein's (1982) Alternating Offers Game. ${ }^{19}$ Even though our approach has desirable properties, it differs from other theories on exchange

17 If the actors perpetually disagree, they do not get a proportion of the surplus. That is, the payoff associated with disagreement is 0 for both network partners.

18 Binmore (1992: 184-188) proves that the solution of the optimization problem specified in eq. (11.1) is the only bargaining solution which satisfies the following three axioms: (A) the bargaining outcome does not depend on how the negotiation partners' utility scales are calibrated; (B) the bargaining outcome is individually rational and Pareto- efficient; (C) the actors' choice is independent of the availability or unavailability of irrelevant alternatives (i.e., if the bargaining partners sometimes agree on a specific outcome when another outcome is feasible, then they will never agree on the latter when the former is feasible).

19 The AOG is a non-cooperative game in which rational egoists alternate in making proposals on how to divide a cake with one time periode elapsing between offers. If the focus is on a scenario in which the amount of time between proposals vanishes, its equilibrium coincides with the generalized Nash bargaining solution (cf., Binmore 1985, 1998; Muthoo 1999; Osborne and Rubinstein 1990). This insight is especially important if one agrees with Nash $(1950,1951,1953)$ and regards non-cooperative games more fundamental than cooperative ones. It is noteworthy that (if theoretical predictions should be tested against experimental results) the subgame perfect Nash equilibrium of the limiting Rubinstein game is robust against deviations from the strict logic of offers and counteroffers as can happen in computerized experiments (Perry and Reny 1993). 
networks. While Bienenstock and Bonacich (1992, 1997) conceptualize a network of potential exchange partners as a cooperative game with transferable utility, they predict negotiation profits via other solutions concepts from cooperative game theory (e.g., core, kernel). Other theorists (e.g., Lovaglia et al. 1995; Skvoretz and Willer 1993) determine the bilateral bargaining outcome by defining and equating "resistance" equations. Although they do not use game-theoretic concepts, resistance theorists (e.g., Willer 1999) emphasize that their approach rests on the premise of strategically rational actors. Still others (e.g., Yamagishi and Cook 1992; Friedkin 1993; Yamaguchi 1996) more or less explicitly refer to non-strategic profitmaximizing behavior when they model exchange relations in networks.

While we think that the foundation in non-cooperative game theory should be a relevant aspect of a theory of network exchange, there are yet other reasons for employing the Nash model over existing theories of sociological exchange theory:

- Bienenstock and Bonacich's (1992, 1997) cooperative solution of the core (or the kernel) neglects the strategic process by which players form coalitions and make demands. From the perspective of non-cooperative game theory, however, it seems necessary to ask for a description of precisely this strategic process. Up to now, the core has no grounding in non-cooperative bargaining theory. From an empirical point of view, on the other hand, it weighs heavy that the core measure does not allow for point predictions for all networks which have so far been experimentally studied. ${ }^{20}$

- Yamaguchi $(1996,2000)$ models the bargaining and exchange game via the assumption of a perfectly competitive market (i.e., an anonymous and centrally located market with many relatively homogenous participants who offer and buy sufficiently homogenous resources only at the systemwide equilibrium price) in which all the price adjustments are costlessly made by a fictitious and neutral Walrasian auctioneer. His approach is a general equilibrium model (viz., each actor acts as if he would solve a constrained CES-utility maximization problem). Hence, Yamaguchi does not allow for strategic rationality, but focusses on dominant strategies only. And, it may be doubted whether this approach is appropriate for the small social settings usually being studied in network exchange experiments.

- A group of prominent models (e.g., Willer and Skvoretz's (1993) ER-model or Lovaglia et al.'s (1995) GPI-RD model) is based on (extensions of) the resistance logic. The resistance logic of these approaches, however, is just an ad hoc transformation of Heckathorn's (1980) original resistance equation with additional but questionable assumptions about the actors' aspiration levels and

20 For example, its application gives only range predictions for a structure as elementary as the 4Line network. Moreover, the core measure predicts unstable exchange outcomes, that is, it has an empty core, for, for instance, the simple Kite structure (see Figure 11.3 for both networks). It thus contradicts experimental work (cf., Willer 1999) which demonstrates just the opposite. 
conflict points. Moreover, these theories do not offer a measure which captures the effects of structural position on bargaining power.

- Heckathorn's (1980) original resistance model, however, corresponds to the KalaiSmorodinsky (1975) bargaining solution (the above mentioned models are not sufficiently characterized by the Kalai-Smorodinsky axioms). Nevertheless, the Nash bargaining solution is to favor over the Kalai-Smorodinsky solution for at least two reasons. First, the latter does not possess a realistic non-cooperative foundation. There is a non-cooperative game invented by Moulin (1984) which is often cited as providing a non-cooperative foundation for the Kalai-Smorodinsky solution. This game, however, has nothing to do with either network exchange experiments nor real life bargaining situations. Among other things, Moulin's game requires the presence of an impartial referee who costlessly organizes consecutive lotteries about feasible proposals in which all the players voluntarily engage. Second, the Kalai-Smorodinsky solution is most relevant in a situation in which the surplus to be divided between two players contracts or expands in a way that possibly makes the set of allocations asymmetric. In network exchange experiments, however, the cakes to be partitioned neither shrink nor grow.

The concrete application of the generalized Nash bargaining solution, even though favorite to competing approaches, requires a numerical specification of each actor's bargaining power. The latter is exogenous to the Nash bargaining model. Therefore, it is precisely here where the basic idea of sociological theories for exchange networks comes in - we assume that, once a relation has been classified as either a rival or a non-rival connection, each actor's bargaining power results from his structural position in the network under consideration. Therefore, our model essentially combines the generalized version of the Nash bargaining solution with a specific definition of each actor's bargaining power in terms of relational features and structural embeddedness.

More precisely, we claim that actors, depending on their structural positions in the exogenous bargaining network and the values of their relations (i.e., the sizes of the cakes to be partitioned), differ in terms of their "network control" (i.e., the extent to which an actor controls the relations to him by his relations to others). And, depending on the actor's assessment of the relation he has with a potential exchange partner (i.e., rival or non-rival), his network control either positively or negatively affects his individual bargaining power. Once individual bargaining powers have been determined, the generalized Nash bargaining solution enshrines the distributions of relative bargaining power and exchange profit in the relations under consideration.

After having introduced the new classification of relations and the generalized Nash bargaining solution, it is left to show how a given negotiation structure determines network control and how it is then related to bargaining power. 


\subsection{Network structure and bargaining power}

Consider an exogenously given bargaining network with a fixed number of structural positions and given values of their respective ties (i.e., the size of the cakes to be partitioned). Such a network may reflect a snapshot of a negotiation situation in real world where cake sizes represent surpluses to be negotiated. A positive surplus may materialize if the buyer's willingness to pay exceeds the seller's reservation price. Or, it simply represents a bargaining structure implemented by the experimenter in a laboratory experiment where each tie refers to a fixed amount of points available for negotiation. Starting from such a network, we postulate that actors, depending on their structural position and the size of the cakes they can negotiate on, differ in terms of their network control. And, depending on an actor's relational assessment of ties to adjacent partners (i.e., rival or non-rival) and the number of exchanges he intends to complete, his network control positively or negatively affects his individual bargaining power. We now successively present these assumptions and their implications in detail.

\subsubsection{Negotiation structure and network control}

\subsubsection{Unequally valued relations}

Let the $n \times n$ matrix $\mathbf{V}$ with main diagonal elements $v_{i i}=0$ for all $i$ and off-diagonal elements $v_{i j} \geq 0$ for all $i \neq j$ represent the exogenously given network of $m$ valued bargaining relations between the $n$ actors. While the relation between the bargaining partners $i$ and $j$ is always symmetric, an actor's relations with distinct partners may differ with respect to the values at stake - the corresponding off-diagonal elements $v_{i j}=v_{j i}$ express whether $i$ and $j$ are bargaining partners and, if so, how large the cake is they can divide. Formally, it holds $v_{i j}=v_{j i}>0$ in the presence of a bargaining relation between $i \neq j$, but $v_{i j}=v_{j i}=0$ in its absence. ${ }^{21}$

Even if matrices of valued adjacencies differ, they may represent the same relational structure. A standardization is thus reasonable. Let $\mathbf{R}$ be the $n \times n$ matrix of standardized actor relations such that $r_{i j}:=\frac{v_{i j}}{\sum_{k=1}^{n} v_{k j}} \geq 0$ for all $i, j$, and $\sum_{k=1}^{n} r_{k j}=1$ for all $j$. That is, $\mathbf{R}$ is the column-stochastic matrix derived from the valued graph. Its off-diagonal element $r_{i j}$ measures $i$ 's fraction of the systemwide valued relations to $j$. In other words, $r_{i j}$ represents $i$ 's degree of "control” over the valued relations to

21 In the basic scenario, each pair of connected actors bargains over the partition of just one cake with a specific size. In a slightly more complicated case, there may be more than just one reciprocated tie between each pair of connected actors in the network (e.g., each dyad can divide two pies per round of negotiated exchanges). If so, the sum of the relevant surpluses determines the offdiagonal elements of matrix $\mathbf{V}$. 
$j$ in the system. Put differently, $r_{i j}$ informs on $i$ 's control over the total of $j$ 's resources available for negotiation. For example, $r_{i j}=0.333$ means that $i$ "controls" one third of $j$ 's resources to be negotiated on. As will become clear below, in a negotiation structure with homogeneous cake sizes, the interpretation of $r_{i j}$ is even more straightforward. It is the reciprocal of the number of $j$ 's negotiation partners. For instance, $r_{i j}=0.333$ means that $j$ has three negotiation partners of which $i$ is one. Regardless of the cake sizes, it holds $0 \leq r_{i j} \leq 1$, where $r_{i j}=0$ indicates that $i$ has no control over $j$ (i.e., absence of a tie between $i$ and $j$ ) and $r_{i j}=1$ reflects that $i$ has complete control over $j$ (i.e., $i$ is $j$ 's only bargaining partner).

However, $r_{i j}$ reveals only part of the available information on the tie between $i$ and $j$. While the $i$-th row of the matrix $\mathbf{R}$ informs about $i$ 's control over each other actor in the system, the $i$-th column of $\mathbf{R}$ informs about the other's control over $i$. That is, $r_{j i}$ denotes $j$ 's control over the valued relations to $i$. Adding up the relevant pairwise elements of $\mathbf{R}$ defines the network control of actor $i$ :

$$
c_{i j}:=\sum_{k=1}^{n} r_{i k} r_{k i} \text { for all } i \text {. }
$$

Put verbally, $c_{i}$ is the degree to which $i$ controls the valued relations to him by his valued relations to others. For example, $c_{i}=3 / 4$ means that actor $i$ controls, via his valued relations to others, 3/4-th of their valued relations to him. The control fraction $c_{i}$ thus may be interpreted as i's 'structural autonomy' as well. ${ }^{22}$ And, given information about i's valued relations and those of his partners, its calculation is straightforward. As a consequence, we do not have to assume that every actor has complete information about the overall shape of the network structure. For the determination of the control distribution in the system, it suffices to postulate that everyone has complete information about his own valued relations and those of his network partners. In this regard, we need not assume fully rational but boundedly rational actors only.

\subsubsection{Equally valued relations}

The weak informational requirements reflect the parsimony of our operationalization of network control. However, there is only one experimental study which systematically varies the size of the cakes to be partitioned in bilateral negotiations (Bonacich and Friedkin 1998; see section 11.5.3). All other laboratory experiments

22 The definition of network control (cf. eq. (11.2)) shows that the $c_{i}$ 's are the positive main diagonal elements of the $n \times n$ matrix $\mathbf{C}:=\mathbf{R R}$. That is, $c_{i}=c_{i i}>0$ for all $i$. Like $\mathbf{R}, \mathbf{C}$ is a column-stochastic matrix. That is, $0<c_{i} \leq 1$ and $1-c_{i}=\sum_{k \neq i} c_{k i} \geq 0$ for all $i$. Since the upper bound of $c_{i}$ is 1 , its complement $1-c_{i}$ measures $i$ 's 'structural dependence' (i.e., the degree to which the other system members affect, via their valued relations to one another, the valued relations to actor $i$ ). 
focus on networks with equally valued relations. That is, each bilateral bargaining session concerns the division of an identical surplus $v_{i j}=v_{j i}=v$ for all $i \neq j$. Given the current lack of evidence on exchange systems with heterogenous cake sizes, it seems useful to demonstrate the simple calculation of $c_{i}$ for networks with equally valued relations (viz., applications in section 11.5.1 and 11.5.2).

Let $v_{i j}=v_{j i}=v$ for all $i \neq j$. The $n \times n$ matrix $\mathbf{V}$ thus reduces to the $n \times n$ adjacency matrix A with main diagonal elements $a_{i i}=0$ for all $i$ and off-diagonal elements $a_{i j} \in\{0,1\}$ for all $i \neq j$ representing the exogenously given and symmetric bargaining relations. More precisely, $a_{i j}$ is a binary measure for the absence or presence of a mutual tie between the actors $i$ and $j$ (i.e., $a_{i j}=a_{j i}$ is coded as 0 or 1 for all $i \neq j$ ).

Let again $\mathbf{R}$ be the $n \times n$ matrix of standardized actor relations such that $r_{i j}:=a_{i j} / \sum_{k=1}^{n} a_{k j} \geq 0$ for all $i, j$, and $\sum_{k=1}^{n} r_{k j}=1$ for all $j$. In the case of equally valued relations, $\mathbf{R}$ is the column-stochastic matrix derived from the adjacencies bearing the properties as described above.

The calculation of $c_{i}$ is then straightforward. A closer look at eq. (11.2) shows that $c_{i}$ may be alternatively expressed as the mean of the $i$-th row in the matrix $\mathbf{R}$. In other words, $i$ 's network control is the mean of $i$ 's control over the systemwide relations to his partners. Formally,

$$
c_{i}=\frac{1}{n_{i}} \sum_{k=1}^{n} r_{i k}=\frac{1}{n_{i}} \sum_{k=1}^{n} \frac{a_{i k}}{m_{k}} \text { for all } i .
$$

where $n_{i}$ denotes the number of $i$ 's negotiation partners and $m_{i}$ the number of $i$ 's ties in the network structure under consideration. ${ }^{23}$ Since the number of positive elements in the $i$-th row in $\mathbf{R}$ is always $n_{i}$, the relevant control distribution can be practically read off from the standardized actor relations.

The number of $i$ 's bargaining partners, $n_{i}$, coincides with the number of positive elements in the $i$-th row of the adjacency matrix A, too. As indicated by the far right-hand side of eq. (11.3), information about $i$ 's network relations and the number of his partners' ties therefore suffices for the computation of $i$ 's network control as well. Put differently, the distribution of network control can be determined when each actor knows his connections and the connections of his partners.

The concept of network control thus requires only weak assumptions about the structural information of network members. This becomes more obvious if $c_{i}$ is

23 In the basic scenario of an adjacency matrix, the number of each actor's negotiation partners $n_{i}$ is equal to the number of his ties $m_{i}$ (i.e., $n_{i}=m_{i}$ for all $i$ ). If, however, more than just one cake per round is to be divided by each pair of connected network members, every actor has more ties than partners (i.e., $m_{i}>n_{i}$ for all $i$ ). For instance, if $i$ has $n_{i}=3$ bargaining partners where two cakes per tie can be divided, we have that $m_{i}=6$. If more than one cake per round can be divided between $i$ and $j$, we assume per definition that $i$ and $j_{n}$ are connected by the respective number of ties. In such a case, we can alternatively write $r_{i j}:=a_{i j} / \sum_{k=1}^{n} a_{k j}=a_{i j} / m_{j} \geq 0$ for all $i, j$. 
expressed in still another way. When $S_{i}$ denotes the set of the $n_{i}$ bargaining partners of actor $i$, it is possible to rewrite eq. (11.3) as follows:

$$
c_{i}=\frac{1}{n_{i}} \sum_{k \in S_{i}} \frac{1}{n_{k}}=\frac{1}{\left(n_{i} / \sum_{k \in S_{i}}\left(1 / n_{k}\right)\right)} \text { for all } i .
$$

Stated differently, $i$ 's network control $c_{i}$ reflects how many negotiation partners actor $i$ and his partners have. Hence, information about the number of $i$ 's bargaining partners and the numbers of their partners allows the calculation of $i$ 's network control. $^{24}$

A closer inspection of the far right-hand side of eq. (11.4) shows, moreover, that the concept of network control is compatible with a rational actor perspective $-c_{i}$ is simply the reciprocal of the estimate a rational actor $i$ will have for the mean number of partners of his partners in a given network. ${ }^{25}$ It thus holds that, for example, $c_{i}=\frac{3}{4}$ expresses that the average number of bargaining partners of $i$ 's partners is $\frac{4}{3}=1.333$. Clearly, $i$ 's network control $c_{i}$ decreases if the mean number of partners of $i$ 's partners increases. And, $c_{i}$ rises if the mean number of bargaining partners of $i$ 's partners falls. In the limit case, the mean number of bargaining partners of $i$ 's partners equals 1 (i.e., actor $i$ is their only negotiation partner) such that $i$ has full network control $c_{i}=1$. So, when the assumption is made that $i$ 's behavior reflects his network control or structural autonomy, it is postulated, in effect, that he takes account of the mean number of partners of his partners.

Whether or not we focus on bargaining structures with equally valued relations, the weak informational requirements reflect that i's network control captures the structure only two steps from $i$ (but neglects structural effects which are three or more steps away). The degrees of network control thus may be seen as parsimonious indicators for the actors' structural positions. They are, by postulate, essential determinants of the individual bargaining powers. That is, actor $i$ 's network control $c_{i}$ affects $b_{i}$, $i$ 's level of individual bargaining power. The direction of the relationship between network control $c_{i}$ and bargaining power $b_{i}$ depends, however, on $i$ 's categorization of the respective network relation.

Referring to the typology of relational orientations introduced in section 11.2, we now can specify how network control affects individual bargaining power for

24 For the determination of the distribution of network control, we thus do not have to assume that every actor has complete information about the overall shape of the network structure. It suffices to postulate that either everyone has complete information about his own relations and his partners' ties or that everyone knows the number of his bargaining partners and the number of the partners' partners.

25 Feld (1991) explains why friends always seem to have more friends than oneself. For the scenario in which this 'class size paradox' is fully understood, he derives the appropriate estimate for the mean number of friends of an individual's friends. The latter corresponds with the denominator of the far right-hand side of eq. (11.4). 
given specific relational orientations. For that purpose, imagine first a system with non-rival relations. Such a network has two essential features: depending on the values involved, $i$ 's bargaining relations are more or less substitutable and compete with the relations of $i$ 's partners to others ("friends of friends are enemies"). ${ }^{26}$ Both features suggest that $i$ 's absolute bargaining power rises with his network control by definition, more control means that $i$ depends less on his current negotiation partners for exchange and that $i$ 's bargaining partners tend to have fewer and/or less valued relations. For the case of rival relations, it thus can be assumed that $i$ 's bargaining power $b_{i}$ rises with i's network control $c_{i}$.

It is reasonable to postulate just the contrary for the opposite scenario of nonrival relations. The features of non-rival relations thus suggest that $i$ 's bargaining power increases if $i$ 's network control decreases. If an actor's structural autonomy is lower, the others more affect, via their valued links to one another, the valued relations to him. Put differently, if his network control is smaller, an individual has, by definition, a higher structural dependence. Especially in the (experimentally relevant) case where different goods flow through the network, this creates additional opportunities in a non-rival setting. Due to the others' transactions, a less autonomous actor may serve as a broker - that is, an actor who either crucially controls the flow of a resource through the system or who controls a specific resource in the system (e.g., a subject who receives one resource for input into the system). ${ }^{27}$ In such a setting, relations with different partners are complementary for the resource flow through the system, and concluded exchanges promote transfers with others. The exchange partners of an individual's partners thus appear as intermediaries ("friends of friends are friends"). For the case of non-rival relations, it thus can be assumed that $i$ 's bargaining power $b_{i}$ rises if $i$ 's network control $c_{i}$ falls.

\subsubsection{Relational assessments and bargaining power}

Drawing on the typology of relational orientations introduced in section 11.2 and taking into account that actors may differ in terms of the number of exchanges they

26 In extremum, this refers to a situation in which bargaining sessions with different partners concern the distribution of just one homogeneous good (what is elsewhere termed a negatively connected network). As a matter of fact, if there are at least two distinct resources, negative connections are also possible - negotiation partners then have to be willing to substitute one good for the other on a one-to-one basis (perfect substitutes).

27 Non-rival relations may be characterized by the property that exchanges with distinct partners increase individual benefits. Such a situation exists, for example, if the focus is on pairwise distributions of different goods which are always consumed together in fixed proportions (perfect complements) - since every actor only cares about the combination of those goods, he will successively engage in a series of dyadic bargaining sessions to obtain as much as he can from each good. Note, however, that non-rival relations do not necessarily involve distinct goods. 
wish to complete, we now show how an actor's bargaining power comes about. To capture the effect of potential variations in the number of desired exchanges across the network, we model two plausible hypotheses: actor $i$ 's bargaining power $b_{i}$ increases (decreases) if, ceteris paribus, $i$ has a rival (non-rival) orientation and wants to complete fewer (more) exchange relations. Put differently, the number of exchanges a position needs to conclude can weaken a rather beneficial network position but can also improve the bargaining power of a rather weak structural position.

In detail, we start from the postulate that the number of desired exchanges $\left(g_{i}\right)$ matters at certain positions more than at others. More precisely, we introduce a case distinction in the following definition of a node-specific weight $z_{i}$ :

$$
z_{i}:=\left\{\begin{array}{l}
g_{i} \text { if } n_{i}=1, \text { or, } g_{i}=1, \text { or, } m_{i}=m, \text { or, } g_{k}=g \text { for all } k, \\
\text { or, } m_{j}=m_{i}, g_{j}=g_{i}, c_{j}=c_{i} \text { for at least one } j \in S_{i}, \\
f_{i}\left(g_{i}\right) \text { otherwise }
\end{array}\right.
$$

where the function $f_{i}\left(g_{i}\right)$ denotes a Box-Cox (1964) transformation of $g_{i}$ :

$$
f_{i}\left(g_{i}\right)=\frac{g_{i}^{d_{i}}-1}{d_{i}} \text { with } d_{i}=1-2 \frac{m-m_{i}}{n(n-1)} .
$$

Put verbally, the node-specific weight $z_{i}>0$ either equals $g_{i}$ or results from a flexible Box-Cox transformation of $g_{i}$ in which, by postulate, the exponent and denominator $d_{i}$ falls as $2\left(m-m_{i}\right) / n(n-1)$ rises. Notice that $z_{i}$ always increases in $g_{i}$, the number of exchanges actor $i$ wants to complete. ${ }^{28}$ Since $2\left(m-m_{i}\right) / n(n-1)$ measures the difference between the network density and $i$ 's density contribution (i.e., the density of the bargaining network without $i$ 's relations), the Box-Cox scenario assumes that the effect of $g_{i}$ on the node-specific weight $z_{i}$ also depends on the number of $i$ 's bargaining relations $\left(m_{i}\right)$, the number of system actors $(n)$, and the number of network relations $(m)$. The combination of these additional parameters reduces the positive effect $g_{i}$ has on the node-specific weight $z_{i}{ }^{29}$

Basically, we postulate that actor $i$ 's bargaining power $b_{i}$ increases with his network control $c_{i}$ when he sees a potential exchange relation as rival. If he classifies a relation as a non-rival one, however, his bargaining power $b_{i}$ decreases with his network control $c_{i}$. To formalize these ideas, we follow Binmore (1985: 273) who defines individual bargaining power as the negative reciprocal of a logarithmic expression.

28 In the Box-Cox scenario, it holds $m>m_{i}>0$ by definition. This ensures $0<d_{i}<1$. Therefore, $f_{i}$ is concave in $g_{i}$ (i.e., $d f_{i} / d g_{i}>0$ and $d^{2} f_{i} / d g_{i}^{2}<0$ ).

29 Since $0<d_{i}<1$ holds in the Box-Cox scenario, $f_{i}$ is always smaller than the exogenously given parameter $g_{i}$. 
Taking into account the node-specific weight $z_{i}$ (and its hypothesized effect on $b_{i}$ ), it is assumed that

$$
b_{i}:=\left\{\begin{array}{ll}
-1 /\left(z_{i} \ln \left(w c_{i}\right)\right), & \text { if } i \text { has a rival orientation } \\
-z_{i} / \ln \left(1-w c_{i}\right), & \text { if } i \text { has a non - rival orientation } \\
-z_{i} / \ln \left(w c_{i}\right), & \text { if } i \text { has a mixed rival orientation } \\
-1 /\left(z_{i} \ln \left(1-w c_{i}\right)\right), & \text { if } i \text { has a mixed non - rival orientation }
\end{array} .\right.
$$

where we use the shorthand

$$
w:=\frac{m+n}{1+m+n}
$$

The latter is a network-specific fraction which rises with the number of mutual ties in the network, $m$, and the number of network members, $n$. In eq. (11.7), the weight $w$ scales the degrees of network control or structural autonomy such that $b_{i}$ is always a positive number. ${ }^{30}$

Equation (11.5) reflects the general character of our approach. All possible network parameters are taken into account in defining an actor's bargaining power. Of course, the additional parameters play no role when the Box-Cox transformation does not apply. By postulate, it holds $z_{i}=g_{i}$ if at least one of the following conditions is met:

- actor $i$ has a peripheral network position (i.e., $n_{i}=1$ );

- actor $i$ wants to complete just one exchange (i.e., $g_{i}=1$ );

- actor $i$ is involved in all relations in a network (i.e., $m_{i}=m$ ), which, in effect, means that $i$ has the central position in a branch structure (i.e., hub-and-spoke network) of arbitrary size;

- actor $i$ belongs to a bargaining network in which each member wants to complete the same number of exchanges (i.e., $g_{k}=g$ for all $k$ );

- actor $i$ faces at least one bargaining partner with the same network control, the same number of bargaining relations, and the same number of desired exchanges (i.e., $c_{j}=c_{i}, m_{j}=m_{i}$, and $g_{j}=g_{i}$ for at least one $j \in S_{i}$ ).

30 An arbitrary choice of $w$ should not be considered since exchange results are of course not robust against choices of the value of $w$. Starting from this insight, there are three reasons for eq. (11.8), the specific definition of $w$. First, admissible transformations of network control are those which just change the unit of scale. The weight $w$ clearly fulfills this requirement. Second, any bargaining network may be characterized by the number of mutual ties, $m$, and the number of system actors, $n$. It thus is reasonable to define the scaling factor $w$ in terms of these system parameters. Third, weighting should preserve the essential role of network control in our approach. The weight $w$ is a systemwide constant which, at most, moderately changes the original values of network control - because of $m \geq 1$ and $n \geq 2$, it holds that $0.75 \leq w<1$. 
These conditions for $z_{i}=g_{i}$ reflect that the application of the Box-Cox transformation does not always make sense. ${ }^{31}$ Their inspection shows that $z_{i}=g_{i}$ is assumed in either limit cases or situations with some form of symmetry. The limit cases include the scenarios in which $i$ intends to complete just one exchange $\left(g_{i}=1\right)$ or resides at an extreme network position $\left(n_{i}=1\right.$ or $\left.m_{i}=m\right)$. The symmetric situations embrace the (experimentally relevant) scenario in which the number of exchanges to be completed does not vary across the network (i.e., $g_{k}=g$ for all $k$ ) as well as the case in which $i$ has at least one fully equivalent bargaining partner $\left(g_{i}=g_{j}\right.$ and $m_{i}=m_{j}$ and $c_{j}=c_{i}$ for at least one $j \in S_{i}$ ). For all other situations, however, we assume that $z_{i}=f_{i}\left(g_{i}\right)$ such that the weight $z_{i}$ increases with the number of desired exchanges $g_{i}$, but always falls short of it.

Starting from the distribution of network control (cf., eq. (11.2)), each actor's bargaining power results from combining eqs. (11.5-11.8) for either relational classification he may have. We then can derive the negotiation outcomes associated with the different types of relations. ${ }^{32}$

\subsubsection{Relational assessments and negotiation outcomes}

If $i$ and $j$ negotiate over the partition of the given cake of size $v_{i j}=v_{j i}$, we assume that they determine their profit shares as if they would solve the optimization problem expressed in eq. (11.1). The maximization of the equivalent welfare function $x_{i j}^{b_{i}}\left(v_{i j}-x_{i j}\right)^{b_{j}}$ implies that $i$ can obtain the exchange profit

$$
x_{i j}=\left(\frac{b_{i}}{b_{i}+b_{j}}\right) v_{i j}=p_{i j} v_{i j} \text { for } i \neq j
$$

31 For example, if $g_{i}=1$, the Box-Cox transformation would not assign a positive value to $z_{i}$. Or, if the number of exchanges to be completed does not vary across the network (i.e., $g_{k}=g$ for all $k$ ), the Box-Cox transformation would not necessarily ensure a common weight $z_{i}=z$.

32 Empirical evidence on exchange networks, by and large, results from experiments in which a systemwide one-exchange rule holds. Subjects can thus conclude only one exchange per round (i.e., $z_{i}=g_{i}=g=1$ for all $i$ ). In such an extreme scenario, eq. (11.7) can be simplified. Substituting $z_{i}=1$ for all cases defining $b_{i}$ in eq. (11.7) facilitates the computation of an actor's bargaining power, such that

$$
b_{i}:=\left\{\begin{array}{l}
-1 / \ln \left(w c_{i}\right), \text { if } i \text { has a rival orientation. } \\
-1 / \ln \left(1-w c_{i}\right), \text { if } i \text { has a non - rival or mixed non - rival orientation. }
\end{array}\right.
$$

Since such negotiation networks show no variation in the allowed number of exchanges, it does not surprise that only network control matters. Consequently, the definition of bargaining power for actors in pure rival (pure non-rival) and mixed rival (mixed non-rival) relations coincides. 
where $p_{i j}:=b_{i} /\left(b_{i}+b_{j}\right)$ defines $i$ 's relative bargaining power in the relation with $j$. And, since $p_{j i}=1-p_{i j}$ holds by definition, $i$ 's partner $j$ will receive $x_{j i}=\left(1-p_{i j}\right) v_{i j}=$ $p_{j i} v_{j i}$. Accordingly, the optimal partition of the given surplus depends critically on the combination of $b_{i}$ and $b_{j}$. Put differently, the bargaining power of just one partner is irrelevant for the negotiation outcome - it is the relative bargaining power (i.e., $p_{i j}$ or $p_{j i}=1-p_{i j}$ ) which matters for the profit split. ${ }^{33}$ Notice, however, that $p_{i j}$ does not coincide with $x_{i j}$ when $v_{i j} \neq 1$. Since $x_{i j}=p_{i j} v_{i j}$ and $x_{j i}=\left(1-p_{i j}\right) v_{i j}$, a comparison of actor $i$ 's profit share with that of his bargaining partner $j$ yields the following chain of equivalent conclusions:

$$
x_{i j} \frac{>}{<} x_{j i} \Leftrightarrow x_{i j} \frac{1}{<} \frac{1}{2} v_{i j} \Leftrightarrow p_{i j} \frac{>}{<} \frac{1}{2} \Leftrightarrow b_{i} \frac{>}{<} b_{j} \text { for } i \neq j
$$

Put verbally, a symmetric distribution of bargaining powers $\left(b_{i}=b_{j}\right.$ or $\left.p_{i j}=1 / 2=p_{j i}\right)$ always yields an equal split of the pie $\left(x_{i j}=v_{i j} / 2=x_{j i}\right)$. There will be an unequal profit division, however, when the power of the two negotiation partners differs. Specifically, $i$ 's exchange profit $x_{i j}$ dominates $j$ 's exchange profit $x_{j i}$ such that $i$ gets more than half of the pie if and only if $p_{i j}$ exceeds $p_{j i}$. Because of $p_{i j}+p_{j i}=1$, the latter is satisfied if and only if $i$ 's relative bargaining power in the relation with $j$ exceeds $1 / 2$. And, this is equivalent to the condition that $i$ 's absolute bargaining power $b_{i}$ exceeds $j$ 's absolute bargaining power $b_{j}$.

The actors' bargaining powers depend, by postulate, on their structural embeddedness and relational classification. As a consequence, the model implications for the distributions of relative bargaining power and surplus in any given match reflects these determinants. Substituting eq. (11.7) into eq. (11.9), we can distinguish four relational types each of which allows specific conclusions about the effects structure has on relative bargaining powers and negotiated exchange profits.

Pure Rival Connection: If the relation between actors $i$ and $j$ is, from their perspective, a pure rival one, then actor $i$ 's profit in match with $j$ is

$$
x_{i j}=p_{i j} v_{i j}\left(\frac{z_{j} \ln \left(w c_{j}\right)}{z_{i} \ln \left(w c_{i}\right)+z_{j} \ln \left(w c_{j}\right)}\right) v_{i j} \text { for } i \neq j
$$

Hence, $i$ 's relative bargaining power and exchange profit in a pure rival connected relation with $j$ rise, everything else being constant, when either $i$ 's network control $c_{i}$ increases or $j$ 's network control $c_{j}$ decreases. Equivalently, $i$ 's relative bargaining

33 As put forward in fn. 24, an actor need not possess complete information about the network as a whole. To calculate $c_{i}$ it is sufficient that he knows his immediate vicinity, that is, the network structure two steps away from him. However, $i$ 's relative bargaining power in match with $j, p_{i j}$, is affected by $c_{i}$ as well as $c_{j}$. This slightly extends the borders of $i$ 's immediate vicinity - to compute his profit share in match with $j, i$ actually requires the knowledge of the network structure three steps away from him. 
power and exchange profit increase, everything else again being constant, ${ }^{34}$ when either $i$ 's number of desired exchanges $g_{i}$ decreases or $j$ 's number of desired exchanges $g_{j}$ increases. $^{35}$

Mixed Rival Connection: If $i$ classifies the relation to $j$ as mixed rival and $j$ categorizes the relation as mixed non-rival, then actor $i$ 's profit in the match with $j$ is

$$
x_{i j}=p_{i j} v_{i j}=\left(\frac{z_{i} z_{j} \ln \left(1-w c_{j}\right)}{\ln \left(w c_{i}\right)+z_{i} z_{j} \ln \left(1-w c_{j}\right)}\right) v_{i j} \text { for } i \neq j .
$$

For such a mixed rival relational orientation, actor $i$ 's relative bargaining power and negotiated profit increase if, everything else being constant, either $c_{i}$ or $c_{j}$ rises. Note that conclusions about a reaction of $p_{i j}$ with respect to a change in $g_{i}$ or $g_{j}$ are only meaningful if the classification of relations always remains unchanged. However, this is not necessarily guaranteed in case of mixed rival, mixed non-rival, and pure non-rival connections.

Mixed Non-Rival Connection: If $i$ classifies the relation to $j$ as mixed non-rival and $j$ categorizes their relation as mixed rival, then actor $i$ 's profit in the match with $j$ is

$$
x_{i j}=p_{i j} v_{i j}=\left(\frac{\ln \left(w c_{j}\right)}{z_{i} z_{j} \ln \left(1-w c_{i}\right)+\ln \left(w c_{j}\right)}\right) v_{i j} \text { for } i \neq j .
$$

In this mixed non-rival relation i's relative bargaining power and profit share increase, everything else being constant, when either $c_{i}$ or $c_{j}$ falls.

Pure Non-Rival Connection: If the relation between actors $i$ and $j$ is, from their perspective, a pure non-rival one, then actor $i$ 's profit in the match with $j$ results from

$$
x_{i j}=p_{i j} v_{i j}=\left(\frac{z_{i} \ln \left(1-w c_{j}\right)}{z_{j} \ln \left(1-w c_{i}\right)+z_{i} \ln \left(1-w c_{j}\right)}\right) v_{i j} \text { for } i \neq j .
$$

Consequently, if their relation is a pure non-rival one, actor $i$ 's relative bargaining power and negotiated profit increase, everything else being constant, if either $i$ 's network control $c_{i}$ falls or $j$ 's network control $c_{j}$ rises.

Since $w:=(m+n) /(1+m+n)$ and $c_{i}:=\sum_{k} r_{i k} r_{k i}$ hold by definition while $r_{i j}$ measures $i$ 's fraction of the systemwide valued relations to $j$, and $g_{i}$ denotes $i$ 's desired

34 These conclusions reflect that $\partial p_{i j} / \partial c_{i}>0, \partial p_{i j} / \partial c_{j}<0$ and $\partial x_{i j} / \partial c_{i}>0$ as well as $\partial x_{i j} / \partial c_{j}<0$. The signs of these partial derivatives inform about the reaction of $i$ 's relative bargaining power $p_{i j}$ and $i$ 's profit share $x_{i j}$ in the match with $j$ when exogenous structural changes affect either $i$ 's network control $c_{i}$ or $j$ 's network control $c_{j}$, but preserve the valued relation between $i$ and $j$.

35 These conclusions reflect that $d p_{i j} / d g_{i}<0, d p_{i j} / d g_{j}>0$ and $d x_{i j} / d g_{i}<0$ as well as $d x_{i j} / d g_{j}>0$. Note, however, that they are only meaningful as long as $m_{i}-g_{i} \geq 2$ as well as $m_{j}-g_{j} \geq 2$ since otherwise the classification of relations changes if $g_{i}$ or $g_{j}$ change. 
number of exchanges, we thus may uniquely predict the distributions of relative bargaining power and negotiated profit for any given combination of relational orientations in each exogenously given bargaining network with $m$ valued ties between $n$ actors. A comparison of the four model conclusions shows, moreover, that the negotiated profits associated with the distinct relational types just differ in terms of $p_{i j}$, $i$ 's relative bargaining power in the match with $j$. And, the calculation of relative bargaining powers and negotiated profits requires, in principle, just a pocket calculator. ${ }^{36}$

Analyses of concrete network structures illustrate, as will become clear below, the straightforward application of our approach. To compare such theoretical predictions with empirical observations, we first need to describe and select relevant experimental studies.

\subsection{Applications}

There are a bulk of experimental results with regard to profit distributions in simple exchange networks (e.g., Bienenstock and Bonacich 1993; Lovaglia et al. 1995; Skvoretz and Fararo 1992; Skvoretz and Willer 1993; Yamagishi, Gillmore, and Cook 1988). However, all these experiments were subject to a relatively homogeneous experimental protocol. Subjects at distinct structural positions did not differ with respect to the number of exchanges they could complete per period. And, there was no variation in the value of the relationships across the network (i.e., equal cake sizes to be divided in all relations). While there are various theoretical approaches (Bienenstock and Bonacich 1992; Burke's 1997; Friedkin's 1986, 1992; Lovaglia et al. 1995; Skvoretz and Willer's 1993; Yamagishi and Cook’s 1992; Yamaguchi’s 1996) which make acceptable predictions for a range of such simple networks (e.g., 4-Line, Stem, Kite, or 3-Branch), there is no general theory of exchange networks which is parsimonious and could coherently be apply to complex network structures. While the

36 Note that dyadically negotiated profits need not always be realized since pure rival networks may reflect exogenous restrictions (e.g., one-exchange rule). This may have further consequences for the network structure as a whole. In such settings (and in mixed exchange networks as well), at least one system member may select his actual exchange partners from a larger set of potential exchange partners. Depending on the negotiation outcomes, he may never complete transfers with one or more of his negotiation partners (consistent or permanent exclusion). Braun and Gautschi (2007) provide an analysis of such "network breaks" and give necessary and sufficient conditions for coincidences or deviations between bargaining and exchange structures. Their conclusions on "network breaks" (e.g., in the T-SHAPE, the H-SHAPE, or the X4-LINE structure) are strongly supported by empirical evidence (viz., Markovsky, Willer, and Patton (1988); Simpson and Willer (1999)). Braun and Gautschi (2007) further provide testable conclusions on a wide range of network structures not yet tested in laboratory experiments. 
Network Control Bargaining (NCB) approach presented in this paper can easily be applied to such simple networks, this paper demonstrates the generality of our model by submitting its theoretical predictions to three different sets of experiments on complex networks.

Due to theoretical considerations and/or experimental evidence on complex networks, some older models have been successively adjusted or revised to now be able to at least address some aspects of complex networks. We compare NCB predictions for dyadic exchange outcomes in complex networks with such alternative approaches. Since none of the alternative approaches makes predictions for both complex characteristics - a variation in the number of exchanges positions can complete per period, and, variations in the value of the relationships across the network -, we refer to different theories for comparison with NCB predictions on these two characteristics.

With regard to results on networks where certain positions were allowed to complete more than one exchange per round, we compare our results to 'updated' versions of Skvoretz and Willer's (1993) GPI-measure. Those are Markovsky et al.'s (1993) GPI-R with an adapted version of the exchange resistance equation, Willer and Skvoretz' (1999) GPI-I-Resistance measure ${ }^{37}$ and Lovaglia et al.'s (1995) GPI-RD measure, however, with a revised interpretation of the degree index. ${ }^{38}$ Empirical validation is done using results from Skvoretz and Willer (1993) as well as from Willer and Skvoretz (1999).

For networks with heterogeneous cake size, we use results from Bonacich and Friedkin (1998). ${ }^{39}$ NCB predictions are compared to Bienenstock and Bonacich's

37 The updated version of the GPI-R measure re-defines (and introduces a case distinction for) the resistance equation. The precise specification of the resistance equation depends on Willer and Skvoretz' (1999) categorization of network connections which draws on the ranking and values of several node-specific parameters. As a consequence, the resulting GPI-R measure is unique to their theoretical approach on network connections (i.e., inclusive, exclusive, null, inclusive-exclusive, and inclusive-null connections; cf., Willer and Skvoretz 1999: 197-199). Moreover, to account for inclusive-exclusive and inclusive-null connections, an additional adjustment in the calculation of the original Graph-theoretic power index (GPI, Markovsky, Willer, and Patton 1988) is necessary. This results in the new GPI-I-Resistance measure.

38 Calculation of the degree index in the original approach is based on the number of ties negotiating partners possess (i.e., their respective number of alternative negotiation partners). However, if a position is allowed (more than) two exchanges per period, the number of negotiation partners is not necessarily unique anymore. A position's effective number of ties need then be calculated as a weighted average of the number of exchange possibilities a position has for each of its allowed exchanges. Consequently, its effective number of ties depends on the sequence of exchanges the position engages in (for an example, see Lovaglia et al. 1995: 142-143).

39 Unfortunately, Bonacich and Friedkin (1998) do not report these results as profit splits between positions, as is normally the case in the literature. Instead, exchange results are graphically depicted. Since the data are not publicly available, we try to read profit splits off these graphics as good as possible. 
(1992, 1997) core measure. This cooperative solution from game theory can be applied rather straightforward to complex networks with differences in the value of the ties if one assumes that the payoff $v(S)$ denotes the maximum value of all cakes the set of actors $S$ in the core can divide. However, there are networks with an empty core. In general, Bonacich and Friedkin suggest to make predictions based on an extension of the Power-Dependence theory (Yamagishi and Cook 1992) which is, under certain assumptions introduced by Bonacich and Friedkin (1998: 162-164), identical to the kernel. Since Friedkin's $(1993,1995)$ Expected-Value theory can also deal with heterogeneous cake sizes, we especially compare NCB predictions to those of these two models. For a concise description of the above mentioned models for exchange networks, we refer the reader to the original literature. Before we move on and confront our theoretical predictions with empirical evidence from complex networks, it is advisable to say a few words about network exchange experiments in general. The design of experiments on exchange networks has common features (see, e.g., Skvoretz and Willer 1991). All experiments consist of several rounds of negotiation and exchange, while the endogenously given relational structure is kept constant. Bargaining sessions involve adjacent network positions only, where usually a cake of identical size (normally 24 "profit points") is to be split in any bilateral match. Experiments generally concern negatively connected networks with a one-exchange rule. That is, the number of exchanges per connection and round is restricted to one. While the latter two points are relaxed in the experiments to be discussed, the experimental protocol otherwise remains unchanged. That also includes that negotiations between adjacent positions occur as a series of offers and counteroffers. Negotiations stop when an agreement is reached. ${ }^{40}$ Bargaining experiments on exchange networks thus reflect the non-cooperative scenario of Rubinstein's (1982) Alternating Offers Game (respectively its limiting solution, see fn. 19). This game has, as this paper made clear, a cooperative solution in the presented generalized Nash bargaining solution (Nash 1950, 1953). ${ }^{41}$

40 Partly due to a computerized setting, proposals can be made within seconds and bargaining sessions do not last long (viz., agreement in less than a few minutes).

41 However, the theoretical assumption of rational and selfish actors need not hold in experimental situations. Student populations need time to learn to rationally play the game, that is, to systematically exploit their strategic position in the network and to maximize exchange profits. Fortunately, Young (1993, 2001: ch. 8) shows that the full rationality assumption is not needed to deduce the generalized Nash bargaining solution. He shows that the generalized Nash bargaining solution is "stochastically stable" (i.e., robustness under small, persistent random shocks (Foster and Young 1990)). That is, the high-rational solution from game theory has a representation in a low-rational environment through the process of learning. Put differently, whether we have hyperrational actors who jump into equilibrium right away or whether boundedly rational actors (who are allowed to make minor mistakes from which they learn) adapt to their environment and learn from past outcomes, does not matter. In equilibrium, both sorts of actors will split the cake as if they would implement the generalized Nash bargaining solution. 
The experimental protocol knows no misdirection. Experimental subjects (normally undergraduates who participate for pay) receive general information about the purpose and the number of rounds of the experiment. They also possess complete information about the bargaining rules, the earnings of their partners, the shape of the negotiation structure, and their own positions within the network. Therefore, experimental results are interpreted as effects the given network structure has on exchange patterns and/or profit divisions between adjacent positions. And, results on exchange profits are represented by the means of profit points the advantaged positions in given matches could realize over several rounds of the experiment. Mostly, this is the mean over the last 10 rounds of the experiment while the first 10 rounds are discharged as learning and adaption rounds.

\subsubsection{Branch-NMQ structures}

Starting point of an exchange network experiment is a specific bargaining structure which limits matches between potential exchange partners. The presentation of experimental findings therefore refers to the types of structural positions (A,B,C,D,E) the different actors have. Individuals located at structurally equivalent positions are normally distinguished by numeric subscripts (e.g., $A_{1}, A_{2}$ ). In this subsection, we exclusively focus on branch structures where a central actor is connected to a specific number of peripheral actors (hub and spoke network). We denote the branch structure under investigation, in accordance with Willer and Skvoretz (1999), as Branch$N M Q$ structures. $N M Q$ refers to node-specific parameters which are, at least partly, unique to their theoretical approach and which are the basis for an alternative categorization of network connections (p. 197-200). $N$ is the number of exchange relations connected to a node (i.e., the number of potential exchange partners), $M$ is the maximum number of relations in which the node can benefit, and $Q$ denotes the minimum number of relations in which exchanges must be completed. ${ }^{42}$ Therefore, $Q$ is a subset of $M$ while the latter itself is a subset of $N$.

$\mathrm{N}$ and $\mathrm{M}$ are thus equivalent to $n_{i}$ and $g_{i}$, respectively, in our theory. Therefore, $(\mathrm{N}-\mathrm{M})$ gives the number of exchange partners which, in every round of bargaining and exchange, are necessarily excluded from exchange. However, since the parameter Q is unique to Willer and Skvoretz' (1999) theory, we cannot compare empirical results for networks with $\mathrm{M} \neq \mathrm{Q}$ to NCB predictions. Take a look at Figure 11.1. NCB makes predictions for Branch311 and Branch333. However, predictions for Branch332 cannot be made by NCB since $M>Q$ (a dotted circle indicates $M=3>2=Q$ ). Only the

42 In network exchange experiments, subjects with $Q<M$ are forced to exchange at least $Q$ times and at most $M$ times. When fewer than $Q$ exchanges with adjacent partners are completed, no points at all, not even from completed exchanges, are paid out. 


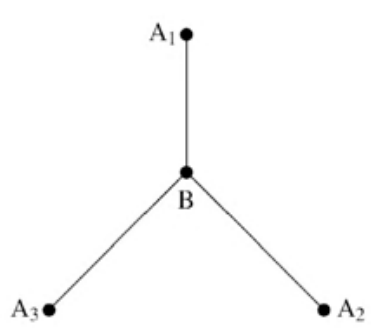

BrANCH 311

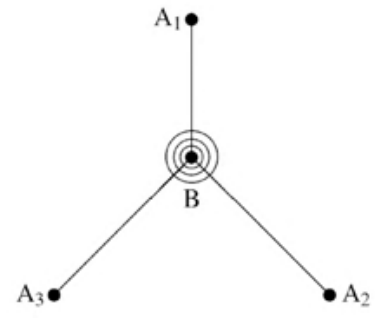

BRANCH 333

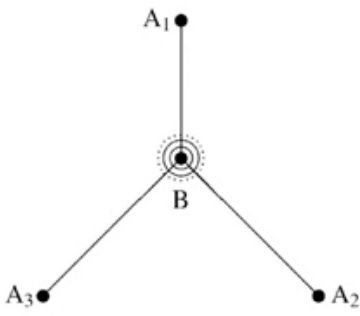

BRANCH 332

Figure 11.1: Example of complex Branch-NMQ structures according to Willer and Skvoretz (1999)

experiment will tell whether the central actor B will eventually conclude two or three exchanges. It thus cannot be decided in advance whether the negotiation structure Branch332 in fact coincidences with a Branch322 or a Branch333 exchange structure. To test NCB predictions against experimental data from Branch-NMQ structures and predictions from GPI-R (Markovsky et al. 1993) with an adapted version of Exchange Resistance, where the definitions of the ER equations depend on the combination of N, M, and Q (see Willer and Skvoretz 1999: 2005), we restrict ourselves to Branch structures where $\mathrm{M}=\mathrm{Q}$ hold. Table 11.1 reports the results.

It can be seen that NCB makes rather accurate predictions for the profit points of $B$ in relation with $A$ in all eight branch structures. Note that the first four branch structures in Table 11.1 are pure non-rival ones while the latter four branch structures are pure rival ones. NCB predictions are less off the empirical observations than predictions from GPI-R in all relations. This is also reflected by the two goodness-of-fit measures we report in Table 11.1.

The branch structure is a network where a central monopolist B can exploit his peripheral exchange partners since he can play the As off against each other (put literally, he can, up to a certain point, threaten the As with permanent exclusion from exchange). It can thus be expected that B's profit increases, ceteris paribus, in the number of exchange partners but decreases, ceteris paribus, in the number of exchanges he must complete. NCB and GPI-R predictions as well as the experimental results clearly show this latter fact. ${ }^{43}$ For instance, the more exchanges B must conclude, the lower his profits, as the comparison of Branch533 to Branch555, or, Branch311 to Branch322 clearly shows. The most dramatic effect, that is, the complete loss of the monopoly rent can be found if $B$ must conclude exchanges with all of the A's (i.e., $\mathrm{N}=\mathrm{M}$ or $n_{i}=g_{i}$ ). In Branch333, B only gets about one third of the profit while in Branch332 he could still get about five sixth of the profit. The same

43 For evidence of the former claim, see Braun and Gautschi (2006). 
Table 11.1: Observed and predicted dyadic profit splits in complex branch structures.

\begin{tabular}{lllrlr}
\hline Network & Match & Tie $^{a}$ & NCB & GPI-R & Observed (s.e.) \\
\hline Branch333 & B:A & pnr & $7.97^{\dagger}$ & $8.92^{\dagger}$ & $7.96(0.61)$ \\
Branch444 & B:A & pnr & $7.37^{\dagger}$ & $8.19^{\dagger}$ & $7.53(0.50)$ \\
Branch555 & B:A & pnr & 6.95 & 7.62 & $5.70(0.34)$ \\
Branch777 & B:A & pnr & 6.39 & 6.82 & $5.09(0.23)$ \\
Branch311 & B:A & pr & $21.65^{\dagger}$ & 23.00 & $21.63(0.49)$ \\
Branch322 & B:A & pr & $19.73^{\dagger}$ & no prediction & $19.62(0.36)$ \\
Branch533 & B:A & pr & 20.80 & no prediction & $19.71(0.30)$ \\
Branch755 & B:A & pr & 20.68 & no prediction & $16.45(0.25)$ \\
\hline AD $^{b}$ & & & $0.55(1.02)$ & 1.33 & \\
MD $^{b}$ & & & $0.36(0.95)$ & 0.63 & \\
\hline
\end{tabular}

Note: Observed profit splits as reported in Skvoretz and Willer (1999). NCB $=$ Network Control Bargaining model. Predictions and observations are for profit points (out of a cake of 24 profit points) of the structural position B. GPI-R (Markovsky et al. 1993) with an adapted version of Exchange Resistance, where the definition of the ER equations depends on the combination of $N, M$, and $Q$ (no predictions for inclusive-exclusive and inclusive-null), see Willer and Skvoretz (1999). ${ }^{a}$ Classification of relations: $\mathrm{pnr}=$ pure non-rival; $\mathrm{pr}=$ pure rival.

${ }^{\mathrm{b}} \mathrm{AD}=$ Absolute Deviation (the sum of absolute distances between observed and predicted profit points relative to number of comparisons); $M D=$ Mean Deviation (the Euclidean distance between observed and predicted profit points relative to number of comparisons). Figures in brackets for NCB refer to the comparison of ties with the latter three networks excluded.

${ }^{\dagger}$ Daggers indicate that predicted values fall within the $95 \%$ confidence interval of the observed values. Put differently, these predictions fit the observations at the $p<0.05$ significance level (twotailed tests).

dramatic collapse in profit points for $B$ can be observed for the remaining $(N=M)$ branches in Table 11.1, that is, for the Branch777 in comparison to the Branch755.

NCB seems to be able to make rather accurate predictions for a first set of complex networks. However, branches are rather simple structures. The following two subsections will use network structures with a little more complexity as the measure rod against to pitch our theory.

\subsubsection{Diamond, $\mathrm{H}$ - and T-Shape structures}

The negotiation networks to be discussed in this subsection basically exhibit the same property as the branch structures just discussed: certain positions must exchange more than once. Figure 11.2 depicts the negotiation networks. For instance, in the H-2-3 network, position $\mathrm{C}$ must conclude two exchanges. He can, however, choose from his exchange partners $A_{1}, A_{2}$, and $D$. On the other hand, position $D$ must exchange three times. Since he only has three possible negotiation partners, he is forced to conclude one exchange per round with each adjacent position. D 


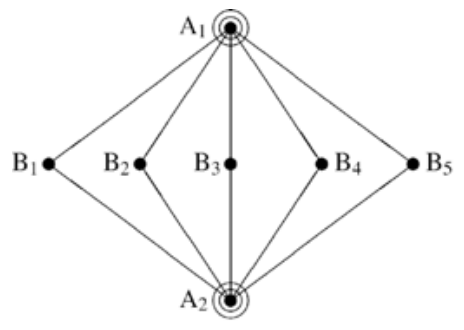

DIAMOND

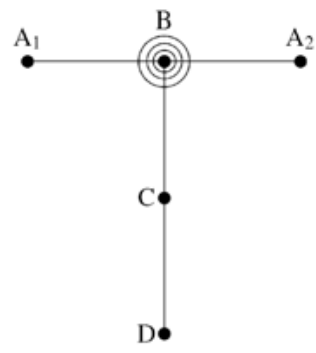

TRIPLE-T

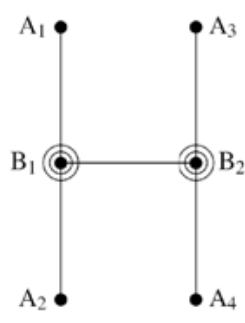

H-2

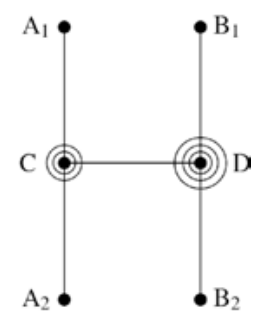

$\mathrm{H}-2-3$

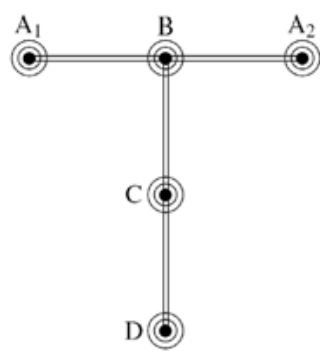

Double-T

Figure 11.2: Examples of complex structures according to Willer and Skvoretz (1999; Diamond, H-2-3) and Skvoretz and Willer (1993, H-2, Triple-T, and Double-T).

thus is in a rather weak negotiation position and it is to be expected that he can be exploited by his partners. Generally, the experimental results should thus show that the advantage of a structural position in a pure or mixed rival relation is weakened by an increasing number of necessary exchanges.

In addition to the number of exchanges per negotiation round, Figure 11.2 introduces yet another feature. A position who must exchange more than once can, but need not, engage in multiple exchanges with an adjacent position. In the Double-T network, the number of lines connecting two adjacent positions indicates the maximum possible exchanges per round between these positions. Position $\mathrm{B}$, who must conclude two exchanges per round, can thus choose to exchange with just one adjacent position ( $A_{1}$ only or $A_{2}$ only) or with two distinct positions (viz., $A_{1}$ and $A_{2}, C$ and $A_{1}$, or $C$ and $A_{2}$ ). While the number of exchanges to be concluded, ceteris paribus, decreases the profit points of the focal positions (the Double-T structure is a pure rival one), it can be expected that the number of cakes which the focal position can divided with one partner, ceteris paribus, increases his bargaining power (and thus his profit points). A position can then threaten more of his partners with permanent exclusion from exchange - due to the possibility of multiple exchanges in one relation - than in a similar situation where only one exchange per connection is possible.

We have seen in subsection 11.5.1, that Willer and Skvoretz (1999) needed to adjust Markovsky et al.'s (1993) original GPI-R measure to cover for networks in which positions differ with respect to the allowed number of exchanges. They do so by 
re-defining (and introducing a case distinction for) the resistance equation. The precise specification of the resistance equation depends on Willer and Skvoretz' (1999) categorization of network connections which draws on the ranking and values of the node-specific parameters N, M and Q. However, as Table 11.2 shows, there are some networks (for which $\mathrm{N}>\mathrm{M} \geq \mathrm{Q}>1$ ) where still no profit point predictions are possible. The application to such, so called, inclusive-exclusive and inclusive-null connections requires - in addition to the updated resistance equations - the adjustment of the original Graph-theoretic power index (GPI; Markovsky, Willer, and Patton 1988) via the "correction index" 1/Q (see for details Willer and Skvoretz 1999:

Table 11.2: Observed and predicted dyadic profit splits in complex diamond, $\mathrm{H}$ - and T-shape structures.

\begin{tabular}{|c|c|c|c|c|c|}
\hline Network & Match & $\mathrm{Tie}^{\mathrm{a}}$ & NCB & GPI-I-R/GPI-RD & Observed (s.e.) \\
\hline Diamond & $A: B$ & $\mathrm{pr}$ & 17.01 & Profit $_{A}=$ Profit $_{A}$ & $18.05(0.23)$ \\
\hline \multirow[t]{3}{*}{$H-2-3$} & $C: A$ & $\mathrm{pr}$ & $18.88^{\dagger}$ & Profit $_{\mathrm{C}: \mathrm{A}}<$ Profit $_{\mathrm{C}: \mathrm{D}}$ & $19.39(0.36)$ \\
\hline & $C: D$ & $\mathrm{mr}$ & $20.77^{\dagger}$ & Profit $_{\mathrm{C}: \mathrm{D}}>$ Profit $_{\mathrm{C}: \mathrm{A}}$ & $20.69(0.48)$ \\
\hline & $\mathrm{D}: \mathrm{B}$ & pnr & $8.38^{\dagger}$ & Profit $_{D: B}>$ Profit $_{D: C}$ & $8.79(0.31)$ \\
\hline $\mathrm{H}-2$ & $B: A$ & $\mathrm{pr}$ & $15.28^{\dagger}$ & 14.60 & $15.50(0.41)$ \\
\hline \multirow[t]{3}{*}{ Triple-T } & $B: A$ & pnr & 7.81 & 12.00 & $13.53(0.45)$ \\
\hline & $\mathrm{B}: \mathrm{C}$ & $\mathrm{mnr}$ & 3.94 & 2.20 & $6.12(1.01)$ \\
\hline & $C: D$ & $\mathrm{pr}$ & 14.64 & $16.00^{\dagger}$ & $17.72(0.93)$ \\
\hline \multirow[t]{2}{*}{ Double- $\mathrm{T}^{\mathrm{b}}$} & $B: A$ & $\mathrm{pr}$ & 20.66 & 19.60 & $20.67(0.49)$ \\
\hline & $C: D$ & $\mathrm{pr}$ & 12.00 & 12.00 & $12.86(0.70)$ \\
\hline$A D^{c}$ & & & $2.01(1.41)$ & 1.67 & \\
\hline$M D^{c}$ & & & $1.15(0.70)$ & 0.81 & \\
\hline
\end{tabular}

Note: Observed profit splits as reported in Willer and Skvoretz (1993) and Skvoretz and Willer (1999). NCB = Network Control Bargaining model. Predictions and observations are for profit points (out of a cake of 24 profit points) of the first named structural position. GPI-I-R makes no point predictions but only compares for (in)equality of profit splits (DIAMOND and $\mathrm{H}-2-3$ ) while GPI-RD allows for point predictions in the remaining networks. However, for GPI-RD, assumptions have to be made with whom a position with more than one possible cake to be divided exchanges first and the number of ties of such an actor are adjusted accordingly (see Lovaglia et al. 1995: 142-143).

${ }^{a}$ Classification of relations: $\mathrm{pr}=$ pure rival; $\mathrm{mr}=$ mixed rival; $\mathrm{pnr}=$ pure non-rival; $\mathrm{mnr}=$ mixed non-rival.

${ }^{\mathrm{b}}$ The Double-T network is not stable in the sense that the negotiation and exchange structure differ (see text for more details).

${ }^{\mathrm{c}} \mathrm{AD}=$ Absolute Deviation (the sum of absolute distances between observed and predicted profit points relative to number of comparisons); $M D=$ Mean Deviation (the Euclidean distance between observed and predicted profit points relative to number of comparisons). Figures in brackets for NCB refer to the comparison of ties of the latter three networks only.

${ }^{\dagger}$ Daggers indicate that predicted values fall within the $95 \%$ confidence interval of the observed values. Put differently, these predictions fit the observations at the $p<0.05$ significance level (twotailed tests). 
215-216). Willer and Skvoretz apply this GPI-I-R model to the Diamond and H-2-3 structures depicted in Figure 11.2. However, no point predictions for profit splits but only relative comparisons are reported (see the first two networks in Table 11.2).

Since we wish to confront NCB predictions with profit point predictions from other theories, we rely on experimental results from H-2, Triple-T, and Double-T (see Figure 11.2) reported in Skvoretz and Willer (1993) and the respective theoretical predictions based on Lovaglia et al.'s (1995) GPI-RD measure. It makes use of a revised interpretation of the degree index, which is part of GPI-RD. Calculation of the degree index in the original approach rests on the relative number of ties negotiating actors possess. However, if a position is allowed to exchange (more than) twice per round, its effective number of ties then depends, by assumption, on the sequence of exchanges the position engages in. To account for this 'path dependency', a position's effective number of ties need to be calculated as a weighted average of the number of exchange possibilities a position has for each of its allowed exchanges (for details see Lovaglia et al. 1995). While GPI-based measures thus can be adapted to embrace situations which deviate from the one-exchange rule, it is unclear how they could account for negotiation structures with heterogeneous cake sizes (see subsection 11.5.3).

Table 11.2 summarizes the empirical results for the networks depicted in Figure 11.2 and in addition reports on the classification of relations for each exchange tie under consideration. First, take a look at the results for the Diamond and H-2-3 structures. GPI-I-R makes no profit point predictions but allows for a ranking comparison of profit splits in different dyads. As can be seen, GPI-I-R predicts the ordinal ranking of profit splits correctly for the H-2-3 networks. Since the As in the Diamond structure are structurally equivalent, it is no surprise that GPI-I-R predicts the same profit shares for the A positions in match with a respective B. NCB calculations, on the other hand, allow for point predictions in both networks which are extremely well in line with empirical observations. For the H-2-3 structure, they all fall within the $95 \%$ confidential interval of the observed values. And, it can clearly be seen that position D in the $\mathrm{H}-2-3$ structure, even though, structurally well positioned, is exploitable because he must conclude one exchange with each of his partners. For the Diamond structure, NCB predicts correctly that the As - even they are forced to conclude two exchanges - are in a far better network position than the Bs. All ties are pure rival ones and the fact that the Bs need to exchange twice only marginally deteriorates their advantageous structural position.

Now take a look at the H-2 structure where the B positions are forced to exchange twice in each round. It can be expected that exchange between $B_{1}$ and $B_{2}$ would result in an equal split of the cake due to their structural equivalence. We know that the simple H-Shape structure with a one-exchange rule (i.e., the Bs can only exchange once in every round) breaks. The bargaining network does not coincidence with the final exchange network since the Bs permanently refrain from exchange with each other and prefer a monopoly position in a 3-Line to exchange with their peripheral As (see Braun and Gautschi 2007). The 3-LINE generates more profit for a rational and profit point maximizing B (almost $84 \%$ of the cake in 
exchange with A) than they could earn in the H-Shape structure (only about $78 \%$ of the cake). The question now arises whether $\mathrm{H}-2$ will show the same property as its simple brother? Since expected profits in pure rival relations decrease in the number of necessary exchanges, B can surely not expect $84 \%$ of the cake in exchange with $A$ in a 3-Line where he must exchange with both As in each round. NCB predicts, for this simple structure, that B could only harvest a little more than $37 \%$ of the cake. However, this is far less than he gets from the As when he at least occasionally exchanges with the other B as well in a H-2 structure. Position B then receives about $64 \%$ of the cake in relation with A (see fifth row in Table 11.2). The H-2 structure therefore is stable in the sense that the negotiation and exchange networks coincide. And, as can be seen, NCB profit point predictions fall within the $95 \%$ confidence interval of the observed values.

We now turn to the Double-T structure where all actors need to complete two exchanges in each round. ${ }^{44}$ Just having in short discussed the logic of network breaks, it is easy understood that the Double-T structure is not stable. The negotiation structure decays into a Double-3-Line and a Double-Dyad due to B's decision to permanently refrain from exchange with $\mathrm{C}$. B can increase his profit in relation with A from about $82 \%$ of the cake in the Double-T to about $86 \%$ of the cake in the Double-3-Line by excluding $\mathrm{C}$ from bargaining and exchange. ${ }^{45}$ The latter is thus forced into a dyad with $\mathrm{D}$ where profit points are split evenly (due to structural equivalence). As Table 11.2 shows, NCB prediction for the Double-T are most accurately in line with laboratory observations.

Finally, let us discuss the Triple-T network. Table 11.2 clearly shows that none of the NCB predictions fall within the 95\% confidence interval. They are far away from observed values, especially for the B:A and B:C relation. To explain this lack of conformity with observed profit splits, take a closer look at the B:A relation. Why should B get about $56 \%$ of the cake in relation with $A$, as experimental findings suggest? $B$ is in a similar situation as in the Branch333 structure (see Figure 11.1) where the observed profit for B in match with A was 7.96 or just about $33 \%$ of the whole cake (see Table 11.1). The crucial argument in favor of NCB predictions now is the fact that in the Triple-T structure, position B even depends on the A's somewhat more since $\mathrm{C}$ no longer is peripheral (as in the branch structure) but itself has a peripheral exchange partner D. This should increase C's negotiation power over B as well as the As negotiation power over B. It can thus be expected that B in relation

44 Note that peripheral actors (i.e., $\mathrm{A}_{1}, \mathrm{~A}_{2}$, and $\mathrm{D}$ ) are not punished if they cannot realize two exchanges in each round. They are dependent on $B$ and $C$, respectively, for negotiation and exchange and those positions dictate whether peripheral actors will indeed realize two exchanges in each round. However, $\mathrm{B}$ and $\mathrm{C}$ must complete two exchanges in each round.

45 Only 4 out of 307 exchanges occurred in the B:C relation. A one-tailed exact binomial test for proportions of exchange shows that this is significantly less than could be expected if positions choose each other at random. 
with A in the Triple-T structure would earn (a little) less than B in relation with A in the Branch333 structure. This is exactly what NCB predicts for the B:A relation: 7.81 profit points in favor of $B$. This is less than the observed 7.96 profit points of $B$ in relation with A in the Branch333 network (see Table 11.1). The observed value of 13.53 profit points in the B:A relation therefore seems puzzling. Personal communication with David Willer, however, confirms our skepticism. The Triple-T experiments were subject to 10 rounds of negotiation and exchange only as well as a small $N$. The empirically observed values for this network, as reported in Table 11.2 should therefore be taken with care.

In sum, one can conclude that NCB predictions for networks where multiple exchanges per round are possible are more than satisfying. We now turn to a final set of networks which again, on the one hand, were subject to a one-exchange rule but then varied the value of the negotiation ties (i.e., varying cake sizes) instead.

\subsubsection{Structures with different cake sizes}

The final set of experiments to be discussed stems from Bonacich and Friedkin (1998) and concerns network structures with unequally valued pure rival relations (heterogeneity with respect to cake sizes). Figure 11.3 depicts the four network structures. Besides the labels for the positions, we now also label the negotiation ties where the respective value of each negotiation tie can be read off the third column in Table 11.3. For instance, there were three different 4-Line structures studied in the experiment. First, one with equal profit points of value 8 in all ties. Second, a 4-Line with cake size 6 in the two B:A relations and a cake size of 12 in the A:A relation. And third, a structure where the B:A relations negotiated over a cake size of 4 and the A:A relation haggled over a cake size of 16.

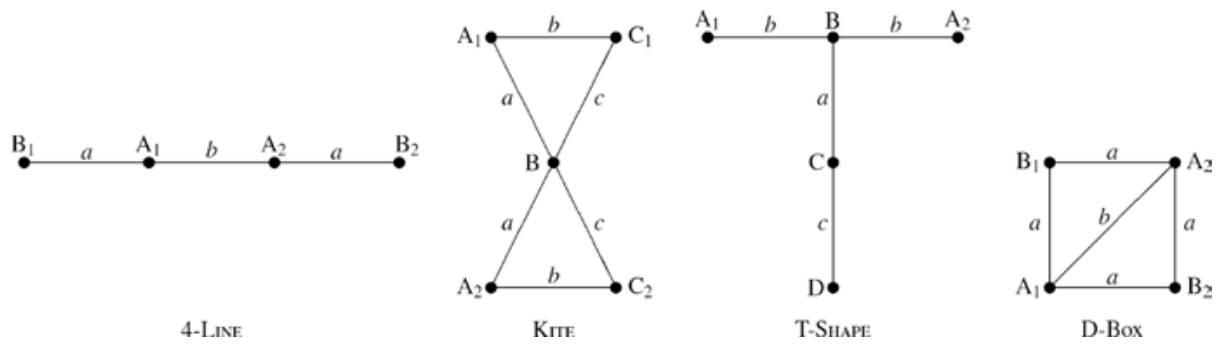

Figure 11.3: Examples of complex structures with varying cake sizes according to Bonacich and Friedkin (1988).

Unfortunately, Bonacich and Friedkin (1998) do not provide point predictions for the experimentally observed profit splits. They do, however, represent the results in 
Table 11.3: Observed and Predicted Dyadic Profit Splits in Complex Structures with Varying Cake Sizes.

\begin{tabular}{llrrrrrrr}
\hline \multicolumn{7}{c}{ Cake Size } \\
\hline Network & Match & a & b & c & NCB & EV & PD & Observed \\
\hline 4-Line & B:A & 8 & 8 & - & 2.70 & 3.04 & 2.72 & 4.00 \\
& & 6 & 12 & - & 1.43 & 2.04 & 0.00 & 2.52 \\
& & 4 & 16 & - & 0.60 & 1.36 & 0.00 & 1.48 \\
Kite & B:A & 8 & 14 & 2 & 2.10 & 1.73 & 0.00 & 3.30 \\
& & 8 & 8 & 8 & 4.62 & 3.47 & 3.47 & 3.60 \\
& & 8 & 2 & 14 & 7.04 & 5.33 & 8.00 & 5.07 \\
T-Shape & B:C & 9 & 6 & 9 & 5.04 & 4.50 & 3.00 & 4.50 \\
& & 12 & 6 & 6 & 7.68 & 6.00 & 6.00 & 6.00 \\
& & 15 & 6 & 3 & 11.10 & 7.50 & 9.00 & 7.50 \\
D-Box & B:A & 8 & 8 & - & 3.46 & 3.36 & 2.00 & 4.00 \\
& & 6 & 12 & - & 2.09 & 2.16 & 0.00 & 2.73 \\
& & 4 & 16 & - & 0.96 & 1.34 & 0.00 & 1.93 \\
\hline AD $^{\text {b }}$ & & & & & 1.27 & 0.43 & 1.76 & \\
MD & & & & & 0.46 & 0.18 & 0.58 & \\
\hline
\end{tabular}

Note: Observed profit splits as graphically depicted in Bonacich and Friedkin (1998).

$\mathrm{NCB}=$ Network Control Bargaining model. Predictions and observations are for profit points of the structural position $\mathrm{B}$ in the relation with value $a$ (cake size). Observed profit points and predictions for the Expected Value theory (EV; Friedkin 1993, 1995) and the Power-Dependence theory (PD; Yamagishi and Cook 1992) are read off Figure 2 in Bonacich and Friedkin (1998: 169) since no table with accurate profit splits is available.

${ }^{\mathrm{b}} \mathrm{AD}=$ Absolute Deviation (the sum of absolute distances between observed and predicted profit points relative to number of comparisons); $M D=$ Mean Deviation (the Euclidean distance between observed and predicted profit points relative to number of comparisons).

graphs (p. 169, Figure 4). The observed profit points reported in Table 11.3 thus are the respective values read off these graphs as accurately as possible. This is also true for the reported predictions of the Power-Dependence theory (Yamagishi and Cook 1992) and the Expected-Value theory (Friedkin 1993, 1995). Bonacich and Friedkin (1998: 162-163) use predictions based on a slight extension of the PowerDependence theory which then is, under certain assumptions, identical to the kernel. No adjustments have to be made to the Expected-Value theory. Bonacich and Friedkin (1998: 169) also "report" profit point predictions by the core. However, since the core is empty for the Kite network and otherwise only makes range predictions, we refrain from reporting and comparing its predictions in detail. It is sufficient to report that NCB predictions and observations for the 4-Line, the T-Shape, and the D-Box fall within the range of the prediction of the core (viz., a share of 0 to 0.5 of the respective cake sizes in the 4-Line and the D-Box, and a share of 0.4 to 0.8 of the whole cake in the T-Shape). 
In networks with equally valued relations, absolute and relative profit shares of $i$ in match with $j$ provide identical information. However, in networks with unequally valued relations, absolute shares can become more important since a small fraction of a large cake can still outperform a large fraction of a small cake. It is therefore to be expected that $i$ 's share in match with $j$, ceteris paribus, increases when the value of relation in the $i: k$ match increases. Since the latter then becomes more favorable to $i$, $j$ 's only chance to be considered for further exchange is to increase his offer to $i$.

Unfortunately, this scenario is not implemented in a "pure form" in the networks under study. However, this mechanism can be observed in the 4-Line. The value of the A:A tie was increased in steps from 8 to 16 while, however, the value of the B:A tie was changed as well. Therefore, we also consider B's relative profit shares in match with A and not just his absolute profit shares. As Table 11.3 shows, B's share of the cake in match with A decreases in absolute and relative terms when the A:A relation increases in value. B gets about 34\% (i.e., 2.70/8) of the B:A cake in an equally valued 4-Line, still about $24 \%$ (i.e., 1.43/6) when the A:A tie is increased in value from 8 to 12 . However, if the A:A value is further increased to 16 , B only receives $15 \%$ (i.e., $0.60 / 4$ ) of the cake in match with $A$ anymore.

While NCB predictions are less accurate than predictions by the Expected Value theory (see goodness-of-fit measures reported in the last two rows of Table 11.3), they are far better than the Power Dependence predictions. And, all theories correctly predict the ordinal ranking of profit splits in the respective networks. Again, recall that observed profit splits as well as those reported for the competing theories have been read off a graphical representation in Bonacich and Friedkin (1998: Table 11.4). These figures are thus subject to some margin of error.

Take a look at the T-Shape structure in Figure 11.3. We have already discussed variants of this structure in the previous subsection. We are interested in the profit splits between B and C (value of relation a). Observations for all three variants of this structure is an equal split of the cake. Expected Value theory predicts precisely this. However, from a structural point of view, this result is not plausible. The structural position $\mathrm{B}$ is always more powerful than $\mathrm{C}$. $\mathrm{B}$ has, in comparison to $\mathrm{C}$, two additional peripheral exchange partners $\left(A_{1}\right.$ and $\left.A_{2}\right)$ who are fully dependent on him for negotiation and exchange. $\mathrm{C}$, on the other hand, only has one additional peripheral partner D. Since bargaining power is a function of the network embeddedness (structural independence in pure rival relations) and $\mathrm{B}$ is better embedded than $\mathrm{C}$, his profit in match with $C$ should exceed half of the pie. B's advantageous structural position is even reinforced by the heterogeneous values of relations - the B:A cake remains constant at $b=$ 6 between experiments while profit points in the C:D relation (value $c$ ) decrease.

In contrast to a T-Shape with equally valued relations which breaks (i.e., no coincidence between the negotiation and exchange structures), NCB now predicts exchanges between $\mathrm{B}$ and $\mathrm{C}$ in all T-Shape structures under investigation. To see this, 
consider the T-Shape where $\mathrm{a}=9, \mathrm{~b}=6$ and $\mathrm{c}=9$. $\mathrm{B}$ receives 4.78 profit points (of $\mathrm{a}$ total of 6) in match with A (not reported in Table 11.3). In an equally valued 3-Line with cake sizes $\mathrm{b}=6$, however, he would receive 4.97 profit points out of 6 (i.e., $p_{B A}=0.8276$, B's relative bargaining profit, times 6). This is more than $\mathrm{B}$ gets from $A$ in the T-Shape. The necessary condition for a network break would thus be fulfilled. But since B gets even more from C, namely 5.05 out of 9, he will keep exchanging with both $\mathrm{A}$ and $\mathrm{C}$. $\mathrm{B}$ thus he has no rational to refrain from permanent exchange with $C$ (as is the case in the simple T-Shape with equally valued relations). And, as discussed in the previous paragraph, B's profit in match with $\mathrm{C}$ exceeds half of the pie - he gets $56 \%$ of the pie from $\mathrm{C}$.

That the T-Shape is stable thus crucially hinges on the fact that the $\mathrm{B}: \mathrm{C}$ relation is valuable to $B$ in absolute terms. Even though in relative terms, B gets less from $C$ if compared to the equally valued T-Shape. In the latter, $p_{B C}=0.6397$ while in the unequally valued T-Shape with $a=9, b=6$ and $c=9, p_{B C}=0.5605$ only. However, since the absolute share of the B:C cake exceeds the absolute share of the $\mathrm{B}: \mathrm{A}$ tie, $\mathrm{B}$ has no incentive to refrain from exchange with $C .{ }^{46}$ The B:C relation should now proof even more valuable when it increases in value. Table 11.3 clearly shows an increase in absolute profit points for B in match with $\mathrm{C}$. And, since the value of the B:A relations (i.e., $b=6$ ) remains constant, it pays less and less for $B$ to break into a 3-Line. Note that since the value of the $\mathrm{B}: \mathrm{C}$ tie increases while (i) the $\mathrm{B}: \mathrm{A}$ remains constant and (ii) the value of the $C: D$ tie decreases, both $B$ and $C$ should become more powerful in match with $\mathrm{A}$ and $\mathrm{D}$, respectively (see discussion above). But because of (ii), $\mathrm{C}$ gets more dependent on $\mathrm{B}$ for a significant absolute share of the exchange profit. Consequently, B's relative power over $C$ also increases from $p_{B C}=0.5605$ in the first T-Shape structure to $p_{B C}=0.6397$ when $a=12$ and, finally, to $p_{B C}=0.7372$ when $a=15$.

As mentioned earlier, figures from Bonacich and Friedkin (1998) should be looked at with some reservation. That the observed profit splits for the T-Shape networks are not especially plausible should increase this skepticism. If we consider the other three networks, NCB predictions could be called reasonably good. Unfortunately, Bonacich and Friedkin (1998) is the only paper which reports observed profit splits from experiments with unequally valued relations. Even though Molm, Peterson, and Takahashi (2001) also provide results on network bargaining in two unequally valued networks, the drawback of their experimental setting was the fact, that they did not put subjects into an alternating offers bargaining setting but only played a five-stage Ultimatum Game. Knowing that NCB predictions assume alternating offers bargaining, we nevertheless in short confront our predictions with their experimental findings.

46 If the value of the B:C relation is 8 or even less (everything else being constant), this logic would no longer hold. In absolute terms, B's share in match with $C$ would then be worse than his share in match with either A. Moreover, B's share in match with either A in the respective 3-Line would outperform his B:A gain in the T-Shape. The T-Shape negotiation structure where $a \leq 8, b=6$ and $c=9$ would therefore break. 
Molm, Peterson, and Takahashi (2001) considered two bargaining networks. First, a Triangle with the $\mathrm{B}: \mathrm{A}$ relation of constant value $\mathrm{a}=16$, the $\mathrm{A}: \mathrm{C}$ relation with constant value $b=4$, and the $B: C$ relation with varying cake sizes $\left(c_{1}=10, c_{2}=16\right.$, and $c_{3}=22$ ). They were interested in changes in power of B over $A$. Since the value of the B: $\mathrm{C}$ tie increases, both $\mathrm{B}$ and $\mathrm{C}$ should become more powerful in relation to $\mathrm{A}$. Therefore, B's profit in relation with A should increase. NCB correctly predicts an increase in B's profit over $\mathrm{A}$ when the $\mathrm{B}: \mathrm{C}$ relation increases in value, starting with 10.28 profit points, then 11.54 profit points, and finally 12.32 profit points (always out of a total of 16 profit points). Experimentally observed values were, respectively, 9.44 profit points, 11.68 profit points, and 12.80 profit points. These values, however, have a rather large standard error such that all NCB predictions fall within the 95\% confidence interval.

The second experiment was conducted using a Box structure (i.e., four actors residing on each corner of a quadrangle). $B_{1}$ is tied to $B_{2}$ (with varying cake sizes of again $c_{1}=10, c_{2}=16$, and $\left.c_{3}=22\right), B_{2}$ is further connected via a relation of a constant value of $a=16$ profit points to $A_{2}$ while $A_{2}$ in turn is tied via a relation of a constant value of $b=4$ profit points to $A_{1}$. Finally, $A_{1}$ "closes the circle" with a relation of a constant value of $a=16$ profit points to $B_{1}$. The line of reasoning is again the same as in the Triangle network. As the $B_{1}: B_{2}$ relation increases in value, $B_{1}\left(B_{2}\right)$ should become more powerful in relation to $A_{1}\left(A_{2}\right)$. Again, NCB correctly predicts the increase in absolute cake sizes. The predicted values of 9.13 profit points, 10.10 profit points, and 10.88 profit points, again out of a total of 16 profit points, fall within the $95 \%$ confidence interval of the observed values of 7.84 profit points, 9.12 profit points, and 10.40 profit points, respectively. Again, relatively large standard errors characterize the observed values.

Whether these large standard errors are due to only five rounds of offers and counteroffers or yet other aspects of the experiment cannot be answered. However, it stands out that NCB predictions are consistently larger than the observed values. It would be interesting to see whether these experimental observations would tend toward the Nash equilibrium if a true alternating offers bargaining situation had been implemented. In general, observations on the development of bilateral negotiations (i.e., offers and counteroffers) until an agreement is reached would show whether experimental subjects ever reach the Nash equilibrium and if so, how fast this occurs. Adjusting exchange theories to be able to make predictions about the evolution of offers and counteroffers over time - and not just about the profit split in the exchange equilibrium - would thus be a desirable next step.

\subsection{Conclusion}

This paper presented an approach to the study of complex bargaining and exchange structures. Partly due to experimental evidence on negatively connected 
exchange networks with a one- exchange rule, model building has widely neglected possibly more complex features of exchange networks. Therefore, only a few theories are capable of making profit point predictions for bargaining and exchange structures where (i) the one-exchange rule is relaxed and positions are allowed to conclude more than one exchange per round, and, (ii) with unequally valued relations across the bargaining structure. However, there is no theory which can be applied to bargaining and exchange structures which are characterized by (i) and (ii). The NCB model seeks to close this gap.

In the course of developing the new model, we have found a weakness in the well-established network classification of negatively and positively connected relations. We therefore introduce a new and more precise classification of network relations which embrace negatively and positively connected ties as special cases. The new network classification is based on structural features and two node specific, but endogenous network parameters: the number of ego's bargaining partners, the number of ego's bargaining relations, and the number of exchanges ego intends to complete. The resulting parsimonious classification allows for unique profit point predictions in networks with combinations of different relational aspects.

In accordance with other theories, our approach reflects the idea that rational actors take advantage of their structural positions in negotiations. Moreover, positions are now aware that the number of exchanges which must be concluded and the value of the respective bargaining ties (i.e., the size of the cakes to be divided in bilateral bargaining) can further deteriorate or improve the (dis)advantage of a specific network position. Contrary to other sociological models, we take into account that negotiation partners pursue their self-interest and thus specify the actors' optimization problem and show where these network parameters enter the choice calculus and how they influence decision making. To comply with this demand we have combined the generalized Nash bargaining solution from game theory with the assumption that both relational features and network positions affect exchange outcomes. The applications section has shown that the resulting Network Control Bargaining (NCB) model makes predictions which closely correspond to experimental results by Bonacich and Friedkin (1998), Molm, Peterson, and Takahashi (2001), Skvoretz and Willer (1993), and Willer and Skvoretz (1999).

While the presented NCB approach can handle different relational features of exchange networks, it still assumes interindividual heterogeneity with respect to characteristics such as age, gender, education or wealth (status). Since it is known that such attributes can matter for negotiation results (e.g., D'Exelle et al. 2010; Dufwenberg and Muren 2006; Eckel and Grossman 2001; Holm and Engsfeld 2005; Schwieren and Sutter 2008; Solnick 2001), a broader approach should account for non-network related characteristics which may influence bargaining outcomes. Such personal variables, $a_{i j}$, could be taken into account via the actors' individual bargaining powers: $b_{i}=f\left(z_{i}, c_{i} ; a_{i j}\right)$. 
Besides these limitations which prevent that the model captures all relevant aspects of real world negotiations, it must be emphasized that the approach rests on strong premises. They ensure that the theoretical model mirrors the bargaining protocol of laboratory exchange networks. However, conditions in laboratory experiments should not be set in stone. It thus would be a worthwhile task to construct less artificial (laboratory) experiments while concurrently broadening the theoretical models for sociological exchange studies.

The behavioral postulate of the model would mark a good point of departure for such a task. According to our theory, each actor is the neoclassical selfish profit maximizer. Even though the experimental protocol tries as good as possible to induce profit point maximizing behavior, it cannot completely suppress additional motivations such as fairness and aversion to inequality. These motivations have been studied widely in the recent literature on behavioral game theory (for an overview and introductions, see Camerer 2003) and shown to be present (e.g., Dictator Game, Ultimatum Game) even in best controlled and artificial laboratory experiments. Likewise, experimental subjects engaging in repeated negotiation and exchange may produce positive feelings towards their exchange partners. They form attachments and make commitments in durable negotiations (e.g., Lawler and Yoon 1996). While some experimental protocols try to inhibit such behavior by rotating subjects through all positions of a network, it only helps to forfend artificial laboratory conditions. An increase in relational cohesion stabilizes exchange relations, breeds trust and is to the benefit of both bargaining partners. These aspects of durable exchange relations deserve attention because of their importance in everyday life.

It is well established through experiments that motivations such as fairness and aversion to inequality unfold equal forces even across different cultural backgrounds (e.g., Camerer 2003; Henrich 2000). Nevertheless, there seem to be norms in distributive bargaining which could further affect outcomes in real world settings (Gautschi 2018). As Young (1996: 116) puts it: “[. . .] norms condition the parties to expect certain outcomes that depend on the bargaining context." Moreover, such norms or convention can even change over time. A realistic model of bargaining and exchange should thus be able to account for the bargaining context. The real world is not the clean laboratory environment with its legitimate tendency to create congeneric situations.

In sum, while the current model is broader in scope and thus capable of making predictions for more complex bargaining and exchange networks, it nevertheless lacks some relevant real world features, as just discussed above. Therefore, the generalization of the current model beyond relational features (such as more than one exchange per round) and unequally valued relations is important and worthwhile. Especially if the model should be suited for the analysis of real bargaining and exchange situations. They are hardly ever characterized by the artificial and highly controlled conditions of a laboratory experiment. 


\section{References}

Aumann, Robert J. and Roger B. Myerson 1988. "Endogenous Formation of Links between Players and Coalitions: An Application of the Shapley Value." Pp. 175-191 in The Shapley Value. Essays in Honor of Lloyd S. Shapley, ed. Alvin E. Roth. Cambridge: Cambridge University Press.

Bala, Venkatesh and Sanjeev Goyal. 2000. "A Noncooperative Model of Network Formation." Econometrica 68: 1181-1229.

Bayati, Mohsen, Christian Borgs, Jennifer Chayes, Yash Kanoria, and Andrea Montanari. 2015. "Bargaining Dynamics in Exchange Networks." Journal of Economic Theory 156: 417-454.

Bienenstock, Elisa Jayne and Phillip Bonacich. 1992. "The Core as a Solution to Negatively Connected Exchange Networks." Social Networks 14: 231-243.

Bienenstock, Elisa Jayne and Phillip Bonacich. 1993. "Game Theory Models for Exchange Networks: Experimental Results.” Sociological Perspectives 36: 117-135.

Bienenstock, Elisa Jayne and Phillip Bonacich. 1997. "Network Exchange as a Cooperative Game." Rationality and Society 9: 37-65.

Binmore, Kenneth G. 1985. "Bargaining and Coalitions." Pp. 269-304 in Game-theoretic Models of Bargaining, ed. Alvin E. Roth. Cambridge: Cambridge University Press.

Binmore, Kenneth G. 1987. “Nash Bargaining Theory II.” Pp. 61-76 in The Economics of Bargaining, eds. by Kenneth G. Binmore and Partha Dasgupta. Oxford: Blackwell.

Binmore, Kenneth G. 1992. Fun and Games: A Text on Game Theory. Lexington, Mass.: D.C. Heath and Company.

Binmore, Kenneth G. 1998. Game Theory and the Social Contract, Vol. 2: Just Playing. Cambridge: MIT Press.

Bonacich, Phillip. 1998. "A Behavioral Foundation for a Structural Theory of Power in Exchange Networks.” Social Psychology Quarterly 61: 185-198.

Bonacich, Phillip. 1999. "An Algebraic Theory of Strong Power in Negatively Connected Networks."Journal of Mathematical Sociology 23: 203-224.

Bonacich, Phillip and Elisa Jayne Bienenstock. 1995. "When Rationality Fails: Unstable Exchange Networks with Empty Cores." Rationality and Society 7: 293-320.

Bonacich, Phillip and Noah E. Friedkin. 1998. "Unequally Valued Exchange Relations." Social Psychology Quarterly 61: 160-171.

Box, G. and D. Cox. 1964. "An Analysis of Transformations." Journal of the Royal Statistical Society Series B 26: 211-264.

Braun, N. and T. Gautschi. 2006. "A Nash Bargaining Model for Simple Exchange Networks." Social Networks 28: 1-23.

Braun, N. and T. Gautschi. 2007. "Who Exchanges With Whom? A Theory of Exchange Ties and Its Application to Simple Networks." Working Paper, University of Munich and Bern.

Burke, Peter J. 1997. “An Identity Model for Network Exchange.” American Sociological Review 62: 134-150.

Camerer, Colin F. 2003. Behavioral Game Theory. Experiments in Strategic Interaction. Princeton: Princeton University Press.

Chakraborty, Tanmoy and Michael Kearns. 2008. "Bargaining Solutions in a Social Network." Pp. 548-555 in 4th International Workshop on Internet and Network Economics, eds. Christos Papadimitriou and Shuzhong Zhang. Berlin: Springer.

Chakraborty, Tanmoy, Michael Kearns, and Sanjeev Khanna. 2009. "Network Bargaining: Algorithms and Structural Results.". Pp 159-168 in Proceedings of the 10th ACM Conference on Electronic Commerce, eds. John Chuang, Lance Fortnow, and Pearl Pu. Stanford, CA: ACM. Coleman, James S. 1973. The Mathematics of Collective Action. Chicago: Aldine. 
Coleman, James S. 1990. Foundations of Social Theory. Cambridge: The Belknap Press of Harvard University Press.

Cook, Karen S. and Richard M. Emerson. 1978. "Power, Equity, and Commitment in Exchange Net works." American Sociological Review 43: 721-739.

Cook, Karen S., Richard M. Emerson, Mary R. Gillmore and Toshio Yamagishi. 1983.

"The Distribution of Power in Exchange Networks: Theory and Experimental Results." American Journal of Sociology 89: 275-305.

Demange, Gabrielle and Myrn H. Wooders. (eds.) 2005. Group Formation in Economics: Networks Clubs, and Coalitions. Cambridge: Cambridge University Press.,

D’Exelle, Ben, Els Lecoutere, and Björn V. Campenhout. 2010. "Social Status and Bargaining when Resources are Scarce: Evidence from a Field Lab Experiment.” Working Paper Series, University of East Anglia, Centre for Behavioural and Experimental Social Science (CBESS) 10-09, School of Economics, University of East Anglia, Norwich, UK.

Dutta, Bhaskar and Matthew O. Jackson. (eds.) 2003. Networks and Groups: Models of Strategic Formation. Berlin: Springer.

Dufwenberg, Martin and Astri Muren. 2006. "Generosity, Anonymity, Gender." Journal of Economic Behavior \& Organization. 61: 42-49.

Eckel, Catherine and Philip Grossman. 2001. "Chivalry and Solidarity in Ultimatum Games.” Economic Inquiry 39: 171-188.

Emerson, Richard M. 1972. "Exchange Theory, Part II: Exchange Relations and Networks." Pp. 58-87 in Sociological Theories in Progress, Vol. 2, eds. Joseph Berger, Morris Zelditch, and Bo Anderson. Boston: Houghton-Mifflin.

Emerson, Richard M. 1981. "Social Exchange Theory.” Pp. 30-65 in Social Psychology: Sociological Perspectives, eds Morris Rosenberg and Ralph H. Turner. New York: Basic Books.

Feld, Scott L. 1991. "Why Your Friends Have More Friends than You Do." American Journal of Sociology 96: 1464-1477.

Foster, Dean and H. Peyton Young. 1990. "Stochastic Evolutionary Game Dynamics." Theoretical Population Biology 38: 219-232.

Friedkin, Noah E. 1986. "A Formal Theory of Social Power.” Journal of Mathematical Sociology 12: 103-126.

Friedkin, Noah E. 1992. "An Expected Value Model of Social Power: Predictions for Selected Exchange Networks." Social Networks 14: 213-229.

Friedkin, Noah E. 1993. “An Expected Value Model of Social Exchange Outcomes.” Pp. 163-193 in Advances in Group Processes, Vol. 10, eds by Edward J. Lawler, Barry Markovsky, Karen Heimer and Jodi O'Brien. Greenwich, CT: JAI Press.

Friedkin, Noah E. 1995. “The Incidence of Exchange Networks.” Social Psychology Quarterly 58: 213-221.

Gautschi, Thomas. 2002. Trust and Exchange. Effects of Temporal Embeddedness and Network Embeddedness on Providing and Dividing a Surplus. Amsterdam: Thela Thesis.

Gautschi, Thomas. 2018. "Risk Aversion and Network Exchange. Experiments on Network Exchange and Heterogeneous Risk Preferences." Working Paper. University of Mannheim.

Gould, Roger V. 2002. "The Origins of Status Hierarchies: A Formal Theory and Empirical Test." American Journal of Sociology 107: 1143-1178.

Heckathorn, Douglas D. 1980. "A Unified Model for Bargaining and Conflict.” Behavioral Science 25: 261-284.

Henrich, Joseph. 2000. "Does Culture Matter in Economic Behavior. Ultimatum Game Bargaining among the Machiguenga of the Peruvian Amazon." American Economic Review 90: 973-979. 
Holm, H. and P. Engsfeld. 2005. "Choosing Bargaining Partners: An Experimental Study on the Impact of Information About Income, Status and Gender." Experimental Economics 8: 183-216.

Jackson, Matthew O. and Asher Wolinsky. 1996. "A Strategic Model of Social and Economic Networks." Journal of Economic Theory 71: 44-74.

Kalai, Ehud and Meir Smorodinsky. 1975. "Other Solutions to Nash's Bargaining Problem." Econometrica 43: 513-518.

Kleinberg, Jon and Éva Tardos. 2008. "Balanced Outcomes in Social Exchange Networks." Pp. 295-304 in Proceedings of the 40th Annual ACM Symposium on Theory of Computing, New York: ACM.

Lawler, Edward J. and Jeongkoo Yoon. 1996. "Commitment in Exchange Relations: Test of a Theory of Relational Cohesion." American Sociological Review 61: 89-108.

Lovaglia, Michael J., John Skvoretz, David Willer and Barry Markovsky. 1995. "Negotiated Exchanges in Social Networks." Social Forces 74: 123-155.

Malinowski, Bronislaw. 1922. Argonauts of Western Pacific. An Account of Native Enterprise and Adventure in the Archipelagoes of Melanesian New Guinea. London: Routledge.

Markovsky, Barry, David Willer and Travis Patton. 1988. "Power Relations in Exchange Networks." American Sociological Review 53: 220-236.

Markovsky, Barry, David Willer and Travis Patton. 1990. "Theory, Evidence and Intuition” (Reply to Yamagishi and Cook). American Sociological Review 55: 300-305.

Markovsky, Barry, John Skvoretz, David Willer, Michael J. Lovaglia and Jeffrey Erger. 1993. "The Seeds of Weak Power: An Extension of Network Exchange Theory." American Sociological Review 58: 197-209.

Markovsky, Barry, David Willer, Brent Simpson and Michael J. Lovaglia. 1997. "Power in Exchange Networks: Critique of a New Theory" (Comment on Yamaguchi 1996). American Sociological Review 62: 833-837.

Molm, Linda D., Gretchen Peterson, and Nobuyuki Takahashi 2001. "The Value of Exchange.” Social Forces 80: 159-184.

Moulin, Hervé. 1984. “Implementing the Kalai-Smorodinsky Bargaining Solution." Journal of Economic Theory 33: 32-45.

Muthoo, Abhinay. 1999. Bargaining Theory with Applications. Cambridge: Cambridge University Press.

Nash, John F. 1950. "The Bargaining Problem.” Econometrica 18: 155-162.

Nash, John F. 1951. "Non-Cooperative Games." Annals of Mathematics 54: 286-295.

Nash, John F. 1953. "Two-Person Cooperative Games." Econometrica 21: 128-140.

Osborne, Martin J. and Ariel Rubinstein. 1990. Bargaining and Markets. San Diego: Academic Press.

Perry, M. and P.J. Reny. 1993. "A Non-cooperative Bargaining Model with Strategically Timed Offers." Journal of Economic Theory 59: 50-77.

Rubinstein, Ariel. 1982. "Perfect Equilibrium in a Bargaining Model." Econometrica 50: 97-109.

Schwieren, Christiane and Matthias Sutter. 2008. "Trust in Cooperation or Ability? An Experimental Study on Gender Differences.” Economics Letters 99: 494-497.

Simpson, Brent and David Willer. 1999. "A New Method for Finding Power Structures.” Pp. 270-284 in Network Exchange Theory, ed. David Willer. Westport, CT: Praeger.

Skvoretz, John and Thomas J. Fararo. 1992. "Power and Network Exchange: An Essay toward Theoretical Unification." Social Networks 14: 325-344.

Skvoretz, John and Michael J. Lovaglia. 1995. "Who Exchanges with Whom: Structural Determinants of Exchange Frequency in Negotiated Exchange Networks." Social Psychology Quarterly 58: 163-177. 
Skvoretz, John and David Willer. 1991. "Power in Exchange Networks: Setting and Structural Variations." Social Psychology Quarterly 54: 224-238.

Skvoretz, John and David Willer. 1993. "Exclusion and Power: A Test of Four Theories of Power in Exchange Networks." American Sociological Review 58: 801-818.

Skyrms, Brian and Robin Pemantle. 2000. "A Dynamic Model of Social Network Formation." Proceedings of the National Academy of Sciences of the United States of America 97: 9340-9346.

Slikker, Marco and Anne van den Nouweland. 2001. Social and Economic Networks in Cooperative Game Theory. Boston, MA: Kluwer.

Solnick, Sara J. 2001. "Gender Differences in the Ultimatum Game." Economic Inquiry 39: 189-200.

Thye, Shane R., Michael J. Lovaglia and Barry Markovsky. 1997. "Responses to Social Exchange and Social Exclusion in Networks." Social Forces 75: 1031-1049.

Vega-Redondo, Fernando. 2007. Complex Social Networks. Cambridge: Cambridge University Press.

Watts, Alison. 2001. "A Dynamic Model of Network Formation." Games and Economic Behavior 34: 331-341.

Willer, David (ed.) 1999. Network Exchange Theory. Westport, CT: Praeger.

Willer, David and Pamela Emanuelson 2008. "Testing Ten Theories." Journal of Mathematical Sociology 32: 165-203.

Willer, David and John Skvoretz. 1999. "Network Connection and Exchange Ratios: Theory, Predictions, and Experimental Tests.” Pp. 195-225 in Network Exchange Theory, ed. David Willer. Westport, CT: Praeger.

Yamagishi, Toshio and Karen S. Cook. 1990. "Power Relations in Exchange Networks: A Comment on 'Network Exchange Theory'." American Sociological Review 55: 297-300.

Yamagishi, Toshio and Karen S. Cook. 1992. "Power in Exchange Networks: A Power-Dependence Formulation." Social Networks 14: 245-266.

Yamagishi, Toshio, Mary R. Gillmore and Karen S. Cook. 1988. "Network Connections and the Distribution of Power in Exchange Networks." American Journal of Sociology 93: 833-851.

Yamaguchi, Kazuo. 1996. "Power in Networks of Substitutable and Complementary Exchange Relations: A Rational Choice Model and an Analysis of Power Centralization.” American Sociological Review 61: 308-332.

Yamaguchi, Kazuo. 1997. "The Logic of the New Theory and Misrepresentations of the Logic" (Reply to Markovsky et al. 1997). American Sociological Review 62: 838-841.

Yamaguchi, Kazuo. 2000. "Power in Mixed Exchange Networks: A Rational Choice Model." Social Networks 22: 93-121.

Young, H. Peyton. 1993. “An Evolutionary Model of Bargaining.” Journal of Economic Theory 59: 145-168.

Young, H. Peyton. 2001. Individual Strategy and Social Structure. An Evolutionary Theory of Institutions Princeton: Princeton University Press. 
